\title{
Three-forms in supergravity and flux compactifications
}

\author{
Fotis Farakos ${ }^{\text {a }}$, Stefano Lanza, Luca Martucci, Dmitri Sorokin \\ Dipartimento di Fisica e Astronomia "Galileo Galilei”, Università degli Studi di Padova \& I.N.F.N. Sezione di Padova, Via F. Marzolo 8 , \\ 35131 Padova, Italy
}

Received: 21 July 2017 / Accepted: 31 August 2017 / Published online: 12 September 2017

(C) The Author(s) 2017. This article is an open access publication

\begin{abstract}
We present a duality procedure that relates conventional four-dimensional matter-coupled $\mathcal{N}=1$ supergravities to dual formulations in which auxiliary fields are replaced by field strengths of gauge three-forms. The duality promotes specific coupling constants appearing in the superpotential to vacuum expectation values of the field strengths. We then apply this general duality to type IIA string compactifications on Calabi-Yau orientifolds with RR fluxes. This gives a new supersymmetric formulation of the corresponding effective four-dimensional theories which includes gauge three-forms.
\end{abstract}

\section{Contents}

1 Introduction . . . . . . . . . . . . . 1

2 Three-form multiplets in supersymmetry . . . . . 3

2.1 Single three-form multiplets . . . . . . . . . 3

2.2 Double three-form multiplets . . . . . . . . . 4

2.3 Double three-form multiplets and non-linear dualization . . . . . . . . . . . . 6

3 Three-form multiplets in $\mathcal{N}=1$ supergravity . . . . 7

3.1 Variant minimal supergravities from duality . . 7

3.1.1 Single three-form supergravity . . . . . . 8

3.1.2 Double three-form supergravity . . . . 9

3.2 Three-form matter-coupled supergravities . . . 9

4 Application to type IIA flux compactifications . . . 11

4.1 Dualization to the three-form effective theory . 12

4.2 Back to the original theory . . . . . . . 13

5 Conclusion . . . . . . . . . . . . . . 14

A Component structure of $\mathcal{N}=1$ superfields . . . 15

B Note on three-forms, scalar potentials and boundary terms ................... 16

C Properties of the Kähler potential and superpotential of type IIA compactifications with RR fluxes . . . . 17

References . . . . . . . . . . . . . . . . 17

a e-mail: fotisfgm@gmail.com

\section{Introduction}

The physical role of gauge three-forms in four-dimensional field theories has been studied for several decades. For instance, constant four-form fluxes of these fields may effect the value of the cosmological constant directly or via couplings of the three-forms to membranes (see e.g. [1-15]). A possible role of three-forms in the solution of the strong $\mathrm{CP}$ problem was discussed e.g. in [16-21] and in inflationary models in [22-27]. In the context of four-dimensional global and local supersymmetric theories, three-form gauge fields can be naturally incorporated as auxiliary fields of supermultiplets, as e.g. in $[8,12-15,28-41]$.

Furthermore, effective field theories with gauge threeforms can find a natural application in the context of string compactifications [25,42-44]. In particular, the effective four-dimensional theories describing flux compactifications of type IIA and IIB string theories should allow for a supersymmetric formulation including gauge three-forms, whose field strengths are dual to the fluxes threading the internal compactified space. In [25] it was suggested that three-forms coming from the dimensional reduction of type II supergravities could be associated with auxiliary fields of chiral and gravity multiplets. However, this idea does not seem to be realizable within any of the four-dimensional supersymmetric models constructed so far.

This problem motivated us to revisit the role of gauge three-forms in four-dimensional rigid and local supersymmetry, focusing on the minimal $\mathcal{N}=1$ case and looking for supergravity-matter models in which the results of [25] could fit. More specifically, we will address the following general question, suggested by the somewhat universal structure of the four-dimensional effective theories describing string flux compactifications. Consider a supersymmetric theory with a set of chiral superfields $\Phi^{A}$ and a superpotential of the form

$W=e_{A} \Phi^{A}+m^{A} \mathcal{G}_{A B}(\Phi) \Phi^{B}+\hat{W}(\Phi)$, 
where $e_{A}$ and $m^{A}$ are real constants, and $\hat{W}(\Phi)$ and $\mathcal{G}_{A B}(\Phi)$ are arbitrary holomorphic functions which, even if not explicitly indicated, can possibly depend on additional chiral superfields. The question is then: does there exist an alternative supersymmetric formulation of the effective theory with a set of pairs of gauge three-forms $\left(A_{3}^{A}, \tilde{A}_{3 A}\right)$ in which the coupling constants $e_{A}$ and $m^{B}$ are promoted to vacuum expectation values of the field strengths $F_{4}^{A}=\mathrm{d} A_{3}^{A}$ and $\tilde{F}_{4 A}=\mathrm{d} \tilde{A}_{3 A}$ ? Note that this procedure is a certain kind of duality transformation that trades coupling constants for gauge three-forms, which do not carry propagating degrees of freedom in four dimensions.

In this paper we will provide a positive answer to this question. The new formulation will be obtained by a supersymmetric duality transformation, which modifies the structure of the chiral multiplets $\Phi^{A}$, substituting their scalar complex auxiliary fields $F^{A}$ or just the real parts thereof with a combination of the field strengths $F_{4}^{A}$ and $\tilde{F}_{4 A}$. Furthermore, this procedure naturally generalizes to the locally supersymmetric case when one of the scalar superfields $\Phi^{A}$ (e.g. $\Phi^{0}$ ) is considered to be the compensator of the super-Weyl-invariant formulation of supergravity. After gauge-fixing the superWeyl symmetry, the duality transformation involves also the auxiliary field of the old minimal supergravity multiplet.

Before arriving at the detailed discussion of the general dualization procedure outlined above, we will first consider the simpler subcases in which $\mathcal{G}_{A B}$ is constant. In these subcases, our dualization explicitly relates the three known types of chiral multiplets: the conventional one with the complex scalar as the auxiliary field, the single three-form multiplet in which the complex auxiliary field is a sum of a real scalar and the Hodge dual of the field strength of a real gauge threeform, and the double three-form multiplet in which the auxiliary field is the field strength of a complex gauge three-form. In particular, the single three-form multiplets arise when the matrix $\operatorname{Im} \mathcal{G}_{\mathrm{AB}}$ is degenerate, as for instance in the extreme case $\operatorname{Im} \mathcal{G}_{\mathrm{AB}} \equiv 0$.

In the case of constant $\mathcal{G}_{A B}$ the relation between the conventional chiral and the dual three-form multiplet is linear. This is no longer true for a general $\mathcal{G}_{A B}(\Phi)$ in which case the duality relation is non-linear and might not allow for a general explicit superfield solution. However, it turns out to be tractable if we assume that $\mathcal{G}_{A B}(\Phi)$ is identified with the second derivative of a homogeneous "prepotential" $\mathcal{G}(\Phi)$ of degree 2. In fact, this is what happens in string flux compactifications.

In the course of the study of the dual formulations with three-form multiplets we will encounter a subtlety regarding the presence of boundary terms in the Lagrangian. The necessity to take into account appropriate boundary terms in the theories with gauge three-forms, either supersymmetric or not, is well known (see e.g. [4,6,7,38]). As we will show, our dualization procedure automatically produces the correct boundary terms, which then do not need to be introduced by hand.

As a concrete non-trivial example, we will perform the duality transformation of the supersymmetric effective theory associated with type IIA orientifold string compactifications on Calabi-Yau spaces with Ramond-Ramond (RR) fluxes. This effective theory has a superpotential of the form (1.1) with $\mathcal{G}_{A B}(\Phi)=\partial_{A} \partial_{B} \mathcal{G}(\Phi)$ and $\mathcal{G}(\Phi)$ being homogeneous of degree 2. In this superpotential the constants $e_{A}$ and $m^{B}$ are identified with the quanta of the internal RR fluxes threading the compactification space and $\Phi^{A}$ with a combination of the Kähler moduli and the super-Weyl compensator superfields. As we will see, the field strengths $F_{4}^{A}$ and $\tilde{F}_{4 A}$ produced by the duality procedure perfectly match the field strengths obtained by direct dimensional reduction of the IIA RR field strengths in [25]. For simplicity, we will work under the assumption that the internal NSNS flux vanishes, which allows us to ignore the tadpole cancellation condition. For more general type IIA, as well as type IIB flux compactifications, the tadpole condition must be appropriately taken into account. Furthermore, the dual formulation with gauge three-forms should allow for a natural incorporation of the open-string sector into the effective theory, as in [42-44]. We leave these interesting developments for the future.

The paper is organized as follows. In Sect. 2 we introduce the duality procedure in rigid supersymmetric theories. We first discuss simpler cases with constant $\mathcal{G}_{A B}$, reviewing the structure of the corresponding known types of chiral three-form multiplets. We then generalize the dualization procedure to a general $\mathcal{G}_{A B}(\Phi)$, which leads to a non-linear duality relation.

In Sect. 3 we extend the duality procedure to supergravity. We first apply it to pure old minimal $\mathcal{N}=1$ supergravity in its super-Weyl-invariant formulation, producing the threeform formulations thereof. In particular, this shows how the different formulations are related to each other by duality transformations of the corresponding super-Weyl compensators. Then we consider models with chiral multiplets coupled to supergravity and apply to them the non-linear duality transformation put forward in the rigid case. The duality acts simultaneously on matter superfields and the super-Weyl compensator. In the resulting dual formulation the auxiliary fields of the chiral and gravity multiplets are expressed in terms of the gauge three-forms and the scalar fields.

In Sect. 4 we apply the duality transformation to the effective four-dimensional theory associated with orientifold type IIA string compactifications with RR fluxes. We also provide the explicit relation between field strengths of the fourdimensional theory and the ten-dimensional RR fields.

In Appendix A we give the component content of the different four-dimensional $\mathcal{N}=1$ superfields which are used in the main text. In Appendix B we show how the dualiza- 
tion procedure works for a simple bosonic field theory and then consider an instructive example which explains how the bosonic boundary terms can be obtained as components of a superspace defined Lagrangian. Appendix C contains useful expressions for the applications to type IIA flux compactifications.

We mainly use notation and conventions of [45].

\section{Three-form multiplets in supersymmetry}

In this section we explain how the dualization procedure works in the case of rigid $\mathcal{N}=1$ supersymmetric theories. In the simplest case of constant $\mathcal{G}_{A B}$ in (1.1), it will produce known variants of off-shell chiral multiplets, whose auxiliary fields are replaced by the field strength of one or two gauge three-forms. We will refer to these chiral multiplets as single and double three-form multiplets, respectively. As we will see, in the case of generic $\mathcal{G}_{A B}(\Phi)$, the dualization will provide a generalization of these off-shell three-form multiplets.

\subsection{Single three-form multiplets}

Consider a rigid supersymmetric theory for a set of chiral superfields

$\Phi^{A}=\varphi^{A}+\sqrt{2} \theta \psi^{A}+\theta^{2} F^{A}$,

with a superpotential of the form (1.1) in the simplest case in which $\operatorname{Im} \mathcal{G}_{\mathrm{AB}}=0$. In such a case, since $\mathcal{G}_{A B}$ is holomorphic, $\operatorname{Re} \mathcal{G}_{A B}$ is necessarily constant and then the Lagrangian takes the form

$\mathcal{L}=\int \mathrm{d}^{4} \theta K(\Phi, \bar{\Phi})+\left(\int \mathrm{d}^{2} \theta\left[r_{A} \Phi^{A}+\hat{W}(\Phi)\right]+\right.$ c.c. $)$,

where $r_{A} \equiv e_{A}+m^{B} \operatorname{Re} \mathcal{G}_{A B}$ are real constants.

To dualize the Lagrangian (2.2), we promote the constants $r_{A}$ to chiral superfields $X_{A}$ and introduce real scalar superfields $U^{A}$ as Lagrange multipliers. The Lagrangian (2.1) gets substituted by

$$
\begin{aligned}
\mathcal{L}^{\prime}= & \int \mathrm{d}^{4} \theta K(\Phi, \bar{\Phi})+\left(\int \mathrm{d}^{2} \theta X_{A} \Phi^{A}+\text { c.c. }\right) \\
& +i \int \mathrm{d}^{4} \theta\left(X_{A}-\bar{X}_{A}\right) U^{A}+\left(\int \mathrm{d}^{2} \theta \hat{W}(\Phi)+\text { c.c. }\right) .
\end{aligned}
$$

Integrating out $U^{A}$ by imposing its equations of motion one gets

$X_{A}-\bar{X}_{A}=0$.
The chirality of $X_{A}\left(\bar{D}_{\dot{\alpha}} X_{A}=0=D_{\alpha} \bar{X}_{A}\right)$ then implies that $X_{A}=r_{A}$, with $r_{A}$ being real constants. Plugging this solution back into (2.3) we get the initial Lagrangian (2.2).

To find the formulation of the theory in terms of threeform multiplets we vary (2.3) with respect to $X_{A}$ subject to the boundary conditions

$\left.\delta X_{A}\right|_{\mathrm{bd}}=0$,

which gives

$\Phi^{A}=Y^{A}$,

with

$Y^{A} \equiv \frac{i}{4} \bar{D}^{2} U^{A}$.

The superfields $Y^{A}$ differ from ordinary chiral superfields only in their $\theta^{2}$-components

$Y^{A}=y^{A}+\sqrt{2} \theta \chi^{A}+\theta^{2}\left({ }^{*} F_{4}^{A}+i D^{A}\right)$,

where $D^{A}$ are real auxiliary scalar fields and

$F_{4}^{A}=\mathrm{d} A_{3}^{A}$.

Hence the real-part of the ordinary scalar auxiliary fields is substituted by the field strengths $F_{4}^{A}$ of the gauge three-form $A_{3}^{A}$, which are part of the $U^{A}$ multiplets (see Appendix A). The three-form fields appear only inside their field strengths because of the invariance of (2.7) under the gauge transformations

$U^{A} \rightarrow U^{A}+L^{A}$,

where $L^{A}$ are arbitrary real linear superfields $D^{2} L^{A}=$ $\bar{D}^{2} L^{A}=0$. This superspace gauge symmetry incorporates the bosonic gauge symmetry

$A_{3}^{A} \rightarrow A_{3}^{A}+\mathrm{d} \Lambda_{2}^{A}$,

and mods out the redundant components of $U^{A}$, which do not survive the chiral projection $\frac{i}{4} \bar{D}^{2}$.

We will refer to the chiral superfields $Y^{A}$ as single threeform multiplets. This kind of scalar multiplet was introduced in [31] and studied in detail in [38]. For instance [38], studied the relation of these multiplets with other multiplets, in particular, with conventional chiral multiplets. The above simple duality argument explicitly shows how the conventional chiral multiplets and three-form multiplets are related in a manifestly supersymmetric way.

To complete the dualization procedure, we should also take into account the equations of motion of $\Phi^{A}$ obtained 
from (2.3) with $\delta \Phi^{A}$ subject to the boundary condition $\left.\delta \Phi^{A}\right|_{\mathrm{bd}}=0$. These give the expression for $X_{A}$ in terms of $Y^{A}$

$X_{A}=\frac{1}{4} \bar{D}^{2} K_{A}(Y)-\hat{W}_{A}(Y)$,

where $K_{A} \equiv \partial_{A} K$ and $W_{A} \equiv \partial_{A} W$.

Upon plugging (2.6) and (2.12) into (2.3) we get the dual Lagrangian describing the dynamics of the superfields $Y^{A}$

$\hat{\mathcal{L}}=\int \mathrm{d}^{4} \theta K(Y, \bar{Y})+\left(\int \mathrm{d}^{2} \theta \hat{W}(Y)+\right.$ c.c. $)+\mathcal{L}_{\text {bd }}$,

where

$\mathcal{L}_{\text {bd }}=i \int \mathrm{d}^{2} \theta\left(\int \mathrm{d}^{2} \bar{\theta}+\frac{1}{4} \bar{D}^{2}\right)\left(\left(\frac{1}{4} \bar{D}^{2} K_{A}-\hat{W}_{A}\right) U^{A}\right)+$ c.c.

is a total derivative and hence a boundary term. Notice that in (2.13) there is no $r_{A} \Phi^{A}$ term in the superpotential. Furthermore, in general the boundary term (2.14) gives a nonvanishing contribution to the Lagrangian and hence cannot be neglected. ${ }^{1}$

The Lagrangian (2.13) has been studied at length in reference [38], to which we refer for further details. In [38] the boundary term has been identified by requiring a consistent variational principle (for previous discussions in nonsupersymmetric settings see e.g. $[4,6])$. On the other hand, the boundary term is automatically produced by our duality procedure, once we fix the form of the Lagrangian (2.3). The only apparent ambiguity, related to the choice of the form $i \int \mathrm{d}^{4} \theta\left(X_{A}-\bar{X}_{A}\right) U^{A}$ of the Lagrange multiplier term in (2.3), is completely fixed by the following criterion: (2.4) must be produced without having to impose specific boundary conditions for the gauge superfield $U^{A}$. Combined with the boundary condition $\left.\delta \Phi^{A}\right|_{\mathrm{bd}}=0$, this implies that in the dual theory (2.13) we need only impose the gauge-invariant boundary condition

$\left.\delta Y^{A}\right|_{\mathrm{bd}}=\left.\frac{i}{4}\left(\bar{D}^{2} \delta U^{A}\right)\right|_{\mathrm{bd}}=0$

As a simple consistency check of the equivalence between the Lagrangians (2.13) and (2.2) we calculate the variation of (2.13) with respect to $U^{A}$, which results in an equation of motion of the form

$\operatorname{Im}\left(-\frac{1}{4} \bar{D}^{2} K_{A}+\hat{W}_{A}\right)=0$.

${ }^{1}$ Note that the Lagrangians (2.3) and (2.13) are gauge invariant under (2.10) provided $X_{A}$ satisfy the boundary conditions $\left.X_{A}\right|_{\text {bd }}=r_{A}$, where $r_{A}$ are (at least, classically) arbitrary real constants which characterize the asymptotic vacuum of the theory. From (2.12) these boundary conditions translate into corresponding boundary conditions for $Y^{A}$.
Combining this equation with the (anti)chirality of its components, it follows that

$-\frac{1}{4} \bar{D}^{2} K_{A}+\hat{W}_{A}=r_{A}$,

where $r_{A}$ can be identified with the real constants appearing in (2.2).

Finally, let us present the explicit form of the bosonic sector of the dual Lagrangian:

$$
\begin{aligned}
\mathcal{L}^{\text {bos }}= & K_{A \bar{B}}\left(D^{A}-i \partial_{m} A^{m A}\right)\left(D^{B}+i \partial_{n} A^{n B}\right) \\
& +\left[i \hat{W}_{A}\left(D^{A}-i \partial_{m} A^{A m}\right)+\text { c.c. }\right]+\mathcal{L}_{\mathrm{bd}}^{\text {bos }},
\end{aligned}
$$

with

$$
\begin{aligned}
\mathcal{L}_{\mathrm{bd}}^{\text {bos }}= & -\partial_{m}\left[i A^{m A}\left(K_{B \bar{A}}-K_{A \bar{B}}\right) D^{B}\right. \\
& \left.+A^{m A}\left(K_{B \bar{A}}+K_{A \bar{B}}\right) \partial_{n} A^{B n}\right] \\
& -\partial_{m}\left(A^{m A} \hat{W}_{A}+A^{m A} \overline{\hat{W}}_{\bar{A}}\right),
\end{aligned}
$$

where $A^{A m} \equiv \frac{1}{3 !} \varepsilon^{m n l p} A_{n l p}^{A}=\left({ }^{*} A_{3}^{A}\right)^{m}$. Notice that the boundary term automatically guarantees a consistent variational principle.

\subsection{Double three-form multiplets}

Let us now consider the dualization of a Lagrangian with a slightly more general superpotential (1.1) in which $\mathcal{G}_{A B}$ is a generic constant matrix and its imaginary part is invertible, $\operatorname{det}\left(\operatorname{Im} \mathcal{G}_{\mathrm{AB}}\right) \neq 0$. Hence we can introduce the arbitrary complex constants

$c_{A} \equiv e_{A}+\mathcal{G}_{A B} m^{B}$,

and rewrite the Lagrangian in the form

$$
\mathcal{L}=\int \mathrm{d}^{4} \theta K(\Phi, \bar{\Phi})+\left(\int \mathrm{d}^{2} \theta\left[c_{A} \Phi^{A}+\hat{W}(\Phi)\right]+\text { c.c. }\right)
$$

As in Sect. 2.1, we can promote the constants to chiral superfields $X_{A}$ by adding appropriate Lagrange multiplier terms to the Lagrangian. The modified Lagrangian is

$$
\begin{aligned}
\mathcal{L}^{\prime}= & \int \mathrm{d}^{4} \theta K(\Phi, \bar{\Phi})+\left(\int \mathrm{d}^{2} \theta\left[X_{A} \Phi^{A}+\frac{1}{4} \bar{D}^{2}\left(\bar{X}_{\bar{A}} \Sigma^{\bar{A}}\right)\right]\right. \\
& \left.+\int \mathrm{d}^{2} \theta \hat{W}(\Phi)+\text { c.c. }\right)
\end{aligned}
$$

where $\Sigma^{\bar{A}}$ are complex linear multiplets, i.e. complex scalar superfields satisfying the constraint

$\bar{D}^{2} \Sigma^{\bar{A}}=0$ 
see Eq. (A.12) for the component expansion of $\Sigma$. This constraint is explicitly solved in terms of a general Weyl spinor superfield $\Psi_{\alpha}^{A}$ as

$\Sigma^{\bar{A}}=\bar{D} \bar{\Psi}^{\bar{A}}$

By integrating out $\Psi_{\alpha}^{A}$ from (2.22) we get the condition $D_{\alpha} X_{A}=0$, which, combined with the chirality of $X_{A}$, implies that

$X_{A}=c_{A}$,

where $c_{A}$ are arbitrary constants. Inserting (2.25) into (2.22) one gets back the Lagrangian (2.21). On the other hand, we can integrate out $X_{A}$ by imposing their equations of motion and get

$\Phi^{A}=S^{A} \equiv-\frac{1}{4} \bar{D}^{2} \bar{\Sigma}^{A}$

where $S^{A}$ are chiral superfields with the following $\theta$ expansion:

$S^{A}=s^{A}+\sqrt{2} \theta \lambda^{A}+\theta^{2 *} G_{4}^{A}$.

Here ${ }^{*} G_{4}^{A}$ are Hodge duals ${ }^{2}$ of the field strengths

$G_{4}^{A}=\mathrm{d} C_{3}^{A}$,

of complex 3-form gauge fields $C_{3}^{A}$. The Hodge duals of $C_{3}^{A}$ are complex vector components of the complex linear superfields $\Sigma^{A}$ (see Appendix A).

We call the chiral superfields $S^{A}$ double three-form multiplets. These kinds of multiplets were introduced in [32] and considered in more detail in [36] but, in contrast to the single three-form multiplets $Y^{A}$ of Sect. 2.1, they have attracted much less attention in the literature. The bosonic gauge transformation $C_{3}^{A} \rightarrow C_{3}^{A}+\mathrm{d} \Lambda_{2}^{A}$ (where $\Lambda_{2}^{A}$ is a complex twoform) are part of the gauge superfield transformation

$\Sigma^{A} \quad \rightarrow \quad \Sigma^{A}+L_{1}^{A}+i L_{2}^{A}$,

where $L_{1}^{A}$ and $L_{2}^{A}$ are real linear superfields.

It is easy to see that (2.29) leaves $S^{A}$ invariant. The counterparts of the gauge transformations (2.29) acting on the 'prepotential' $\Psi_{\alpha}$ are

$\Psi_{\alpha}^{A} \rightarrow \Psi_{\alpha}^{A}+\Lambda_{\alpha}^{A}+D^{\beta} \Lambda_{\beta \alpha}^{A}$,

where $\bar{D}_{\dot{\beta}} \Lambda_{\alpha}^{A}=0$ and $\Lambda_{\beta \alpha}^{A}=\Lambda_{\alpha \beta}^{A}$.

${ }^{2}$ In our conventions, the four-dimensional Hodge dual of a $p$-form $\omega$ is defined by $\left({ }^{*} \omega\right)_{m_{1} \ldots m_{4-p}}=\frac{1}{p !} \varepsilon_{m_{1} \ldots m_{4-p} n_{1} \ldots n_{p}} \omega^{n_{1} \ldots n_{p}}$, where $\varepsilon^{0123}=$ $-\varepsilon_{0123}=1$.
Note that the Lagrange multiplier term in (2.22) is singled out by a criterion analogous to the one introduced at the end of Sect. 2.1. Namely, it leads to (2.25) without the need for any specific boundary condition on the gauge superfield $\Psi_{\alpha}^{A}$ and it directly gives back the original Lagrangian, without involving possible boundary terms. As a consequence, the dual Lagrangian describing the dynamics of the superfields $S^{A}$ is also completely fixed, including the appropriate boundary term. Indeed, by plugging (2.26) back into (2.22), we get the dual Lagrangian

$\hat{\mathcal{L}}=\int \mathrm{d}^{4} \theta K(S, \bar{S})+\left(\int \mathrm{d}^{2} \theta \hat{W}(S)+\right.$ c.c. $)+\mathcal{L}_{\text {bd }}$,

where the boundary term is given by the following total derivative contribution to the Lagrangian:

$\mathcal{L}_{\text {bd }}=\frac{1}{4}\left(\int \mathrm{d}^{2} \theta \bar{D}^{2}-\int \mathrm{d}^{2} \bar{\theta} D^{2}\right)\left(\bar{X}_{\bar{A}} \Sigma^{\bar{A}}\right)+$ c.c.

In (2.32) $X_{A}$ should be replaced by its expression obtained from (2.22) as the equation of motion of $\Phi^{A}$, namely

$X_{A}=\frac{1}{4} \bar{D}^{2} K_{A}-W_{A}$.

An example of the component field form of the boundary term which one gets from (2.32) is given in Appendix B. ${ }^{3}$

Let us now turn to the case of constant $\mathcal{G}_{A B}$ with noninvertible imaginary part $\operatorname{Im} \mathcal{G}_{\mathrm{AB}}$. If $A, B=1, \ldots, n$, then the matrix $\operatorname{Im} \mathcal{G}_{\mathrm{AB}}$ has a rank $r<n$. This implies that there are $n-r>0$ vectors $u_{a}^{A}, a=1, \ldots, n-r$, such that $\operatorname{Im} \mathcal{G}_{\mathrm{AB}} \mathrm{u}_{\mathrm{a}}^{\mathrm{B}}=0$. We can complete them with $r$ vectors $v_{q}^{A}$, $q=1, \ldots, r$, which together with $u_{a}^{A}$ form a basis of $\mathbb{R}^{n}$. We can use this basis to re-organize the chiral superfields as follows:

$\Phi^{A}=\Phi^{a} u_{a}^{A}+\Phi^{q} v_{q}^{A}$

and, analogously, $m^{A}=m^{a} u_{a}^{A}+m^{p} v_{p}^{A}$. Then the superpotential (1.1) takes the form

$W=r_{a} \Phi^{a}+c_{p} \Phi^{p}+\tilde{W}(\Phi)$

where

$r_{a} \equiv\left(e_{A}+m^{B} \operatorname{Re} \mathcal{G}_{A B}\right) u_{a}^{A}, \quad c_{p} \equiv e_{A} v_{p}^{A}+m^{q} v_{q}^{A} \mathcal{G}_{A B} v_{p}^{B}$

3 The free Lagrangian $\hat{\mathcal{L}}_{\text {free }}=\int \mathrm{d}^{4} \theta S \bar{S}$ was briefly discussed in [32]. The component form of (2.31) with $K=\delta_{A \bar{B}} S^{A} \bar{S}^{\bar{B}}$ and $\hat{W}(S)=$ $m_{A B} S^{A} S^{B}+g_{A B C} S^{A} S^{B} S^{C}$ but without the boundary term was considered in [36]. 
are, respectively, arbitrary real and complex constants and

$\tilde{W} \equiv \hat{W}+m^{a} u_{a}^{A} \mathcal{G}_{A B} v_{p}^{B} \Phi^{p}$

We can then proceed by dualizing $\Phi^{a}$ to single three-form multiplets $Y^{a}$ as in Sect. 2.1 and $\Phi^{q}$ to double three-form multiplets $S^{q}$ as in the present section. ${ }^{4}$

\subsection{Double three-form multiplets and non-linear dualization}

We are now ready to consider the more general case of nonconstant holomorphic matrix $\mathcal{G}_{A B}(\Phi)$, still in the case of rigid supersymmetry. Even though not explicitly indicated, the following discussion allows for the inclusion of additional chiral multiplets in the theory, which can enter $\mathcal{G}_{A B}(\Phi)$ and $\hat{W}(\Phi)$ in (1.1), but which are not subject to the dualization procedure. For instance, extra chiral multiplets $T^{p}$ will explicitly appear in Sect. 4, in which we will apply our construction to type IIA flux compactifications.

For convenience we define the matrices

$\mathcal{N}_{A B}=\operatorname{Re} \mathcal{G}_{A B}, \quad \mathcal{M}_{A B}=\operatorname{Im} \mathcal{G}_{\mathrm{AB}}$.

We will assume that, for generic values of the chiral fields $\Phi^{A}$, the matrix $\mathcal{M}_{A B}$ is invertible. We will briefly come back to the degenerate case $\operatorname{det}\left(\mathcal{M}_{A B}\right)=0$ at the end of the section. Furthermore, for simplicity, we assume that $\mathcal{G}_{A B}(\Phi)$ is symmetric, although most of the discussion holds for nonsymmetric $\mathcal{G}_{A B}(\Phi)$. This symmetry is automatic if we regard $\mathcal{G}_{A B}(\Phi)$ as the second derivative of a holomorphic prepotential $\mathcal{G}(\Phi)$, as we will assume in the local supersymmetry case.

Our starting point is the Lagrangian

$$
\begin{aligned}
\mathcal{L}= & \int \mathrm{d}^{4} \theta K(\Phi, \bar{\Phi}) \\
& +\left(\int \mathrm{d}^{2} \theta\left[e_{A} \Phi^{A}+m^{A} \mathcal{G}_{A B}(\Phi) \Phi^{B}+\hat{W}(\Phi)\right]+\text { c.c. }\right) .
\end{aligned}
$$

The strategy followed in the previous sections is then generalized by replacing (2.39) with the following Lagrangian:

$$
\begin{aligned}
\mathcal{L}^{\prime \prime}= & \int \mathrm{d}^{4} \theta K(\Phi, \bar{\Phi})+\left(\int \mathrm{d}^{2} \theta\left[X_{A} \Phi^{B}+\hat{W}(\Phi)\right]+\text { c.c. }\right) \\
& -\frac{1}{4}\left(\int \mathrm{d}^{2} \theta \bar{D}^{2}\left[\Sigma_{A} \mathcal{M}^{A B}\left(X_{A}-\bar{X}_{A}\right)\right]+\text { c.c. }\right)
\end{aligned}
$$

\footnotetext{
4 Notice that the choice of the vectors $u_{a}^{A}$ is not unique, as we could redefine $v_{q}^{A} \rightarrow v_{q}^{A}+\alpha_{q}^{a} u_{a}^{A}$ with $\alpha_{q}^{a}$ being arbitrary real constants. This ambiguity induces the redefinitions $\Phi^{a} \rightarrow \Phi^{a}-\alpha_{q}^{a} \Phi^{q}$ and $c_{q} \rightarrow$ $c_{q}+\alpha_{q}^{a} r_{a}$, which mix the two kinds of dual three-form multiplets.
}

where $\mathcal{M}^{A B}$ is the inverse of $\mathcal{M}_{A B}$ and $\Sigma_{A}=\bar{D} \bar{\Psi}_{A}$ are complex linear superfields defined by Eqs. (2.23) and (2.24).

The extremization of (2.40) with respect to $\Psi_{A}^{\alpha}$ gives

$D_{\alpha}\left(\mathcal{M}^{A B} \operatorname{ImX} X_{\mathrm{B}}\right)=0$

Notice that the variation of (2.40) with respect to $\Psi^{A}$ does not involve any boundary terms and the Lagrange multiplier term in (2.40) satisfies the criterion discussed in the previous sections. The general solution of (2.41) is

$X_{A}=e_{A}+\mathcal{G}_{A B}(\Phi) m^{B}$

with $e_{A}$ and $m^{B}$ being arbitrary real constants. ${ }^{5}$ Hence, by plugging (2.42) back into (2.40) one obtains the original Lagrangian (2.39).

Alternatively, we get the dual description by integrating out $X_{A}$ in (2.39). This results in the following expression for the chiral superfields $\Phi^{A}$ :

$\Phi^{A}=S^{A}$

where

$S^{A} \equiv \frac{1}{4} \bar{D}^{2}\left[\mathcal{M}^{A B}\left(\Sigma_{B}-\bar{\Sigma}_{B}\right)\right]$.

The chiral superfields $S^{A}$ provide a generalization of the double three-form multiplets encountered in Sect. 2.2. Note that, once we impose (2.43), $\mathcal{M}^{A B}$ depends on $S^{A}$. Then, in general, Eq. (2.44) is non-linear and cannot be explicitly solved for $S^{A}$ as a function of $\Sigma_{A}$. However, this does not necessarily create complications in specific applications, as for instance to type IIA flux compactifications discussed in Sect. 4.

The above formulation in terms of $\Sigma_{A}$, which contains gauge three-forms, is invariant under the following gauge transformations which generalize (2.29):

$\Sigma_{A} \rightarrow \Sigma_{A}+\tilde{L}_{A}+\mathcal{G}_{A B} L^{B}$

where $\tilde{L}_{A}$ and $L^{B}$ are arbitrary real linear superfield parameters. This gauge symmetry guarantees that the gauge threeforms enter (2.43) via their gauge-invariant field strengths only. We will discuss the component structure of the relation (2.44) in the supergravity case in Sect. 3.2.

If we substitute the solution (2.43) back into the Lagrangian (2.40) we obtain

$\hat{\mathcal{L}}=\int \mathrm{d}^{4} \theta K(S, \bar{S})+\left(\int \mathrm{d}^{2} \theta \hat{W}(S)+\right.$ c.c. $)+\mathcal{L}_{\text {bd }}$,

$\overline{5 \text { Indeed, from }}$ (2.41) and its complex conjugate one gets $\mathcal{M}^{A B} \operatorname{Im} X_{\mathrm{B}}=$ $\mathrm{m}^{\mathrm{A}}$, with $m^{A}$ being arbitrary real constants. We can then write $X_{A}=$ $\operatorname{Re} X_{A}+i \operatorname{Im} \mathcal{G}_{A B} m^{B} \equiv \operatorname{Re}\left(X_{A}-\mathcal{G}_{A B} m^{B}\right)+\mathcal{G}_{A B} m^{B}$. This equation is compatible with the chirality of $X_{A}$ and $\mathcal{G}_{A B}$ only if $\operatorname{Re}\left(X_{A}-\right.$ $\left.\mathcal{G}_{A B} m^{B}\right)=e_{A}$ are constant. We thus arrive at (2.42). 
where the boundary term is now given by the total derivative contribution

$$
\mathcal{L}_{\text {bd }}=\int \mathrm{d}^{2} \theta\left(\int \mathrm{d}^{2} \bar{\theta}+\frac{1}{4} \bar{D}^{2}\right)\left(X_{A} \mathcal{M}^{A B}\left(\Sigma_{B}-\bar{\Sigma}_{B}\right)\right)+\text { c.c. }
$$

in which $X_{A}$ is expressed via $\Sigma_{A}$ on account of the equation of motion of $\Phi^{A}$, as in Sect. 2.2. We will give the explicit expression of the boundary term in the supergravity case in the next section.

The Lagrangian (2.31) provides us with the dual formulation of the considered theory in terms of the double three-form multiplet (2.44), with the 'reduced' superpotential $\hat{W}(S)$ and the appropriate boundary term. The information as regards the form of the matrix $\mathcal{G}_{A B}(\Phi)$ appearing in the superpotential of the original theory is encoded in the form of the matrix $\mathcal{M}^{A B}$ which enters the definition (2.44) of the double three-form multiplet. On the other hand, as in the previous sections, the constant parameters $e_{A}$ and $m^{A}$ got dualized into the expectation values of the field strengths of the gauge three-forms.

Before passing to the locally supersymmetric case, let us briefly discuss the situation in which $\operatorname{Im} \mathcal{G}_{A B}$, with $A, B=$ $1, \ldots, n$, is degenerate of rank $r<n$. Then there should exist $n-r>0$ real vectors $u_{a}^{A}, a=1, \ldots, n-r$, such that $\operatorname{Im} \mathcal{G}_{A B}(\Phi) u_{a}^{B}=0$ and hence $\mathcal{G}_{A B}(\Phi) u_{a}^{B}=\overline{\mathcal{G}}_{A B}(\bar{\Phi}) u_{a}^{B}$. Taking into account the holomorphicity of $\mathcal{G}_{A B}(\Phi)$, this condition is quite strong and puts strong constraints on the form of $\mathcal{G}_{A B}(\Phi)$. Suppose, for instance, that the vectors $u_{a}^{A}$ are constant, as at the end of Sect. 2.2. This would imply that $\mathcal{G}_{A B}(\Phi) u_{a}^{A}$ is constant too. We could then proceed as in Sect. 2.2, rewriting the superpotential as follows:

$W=r_{a} \Phi^{a}+\left[e_{p}+m^{q} \mathcal{G}_{q p}(\Phi)\right] \Phi^{p}+\hat{W}^{\prime}(\Phi)$,

where $e_{a} \equiv e_{A} u_{a}^{A}, \mathcal{G}_{q p} \equiv u_{q}^{A} \mathcal{G}_{A B} u_{p}^{B}, r_{a}$ is as in (2.36) and $\hat{W}^{\prime}$ is as in (2.37). One can then dualize $\Phi^{a}$ to single threeform multiplets $Y^{a}$ and $\Phi^{p}$ to double three-form multiplets $S^{p}$. We expect similar combinations of different dualizations to be possible in more general cases.

\section{Three-form multiplets in $\mathcal{N}=1$ supergravity}

We now extend the duality procedure described in Sect. 2 for rigid supersymmetry to matter-coupled $\mathcal{N}=1$ supergravity. The extension is rather natural if we use a super-Weylinvariant approach [46]. Before proceeding let us recall that the old minimal formulation of supergravity [28] describes the interactions of the gravitational multiplet

$e_{a}^{m}, \quad \psi_{m}^{\alpha}, \quad b_{a}, \quad M$
The physical fields are the vielbein $e_{a}^{m}$ and the gravitino $\psi_{m}^{\alpha}$, whereas the auxiliary fields are the real vector $b_{a}$ and the complex scalar $M$.

We will construct three-form matter-coupled supergravity by dualizing a super-Weyl-invariant formulation. The curved superspace super-vielbeins transform as follows under the super-Weyl transformations [46]:

$$
\begin{aligned}
E_{M}^{a} \rightarrow \mathrm{e}^{\Upsilon+\bar{\Upsilon}} E_{M}^{a}, \\
E_{M}^{\alpha} \rightarrow \mathrm{e}^{2 \bar{\Upsilon}-\Upsilon}\left(E_{M}^{\alpha}-\frac{i}{4} E_{M}^{a} \sigma_{a}^{\alpha \dot{\alpha}} \overline{\mathcal{D}}_{\dot{\alpha}} \bar{\Upsilon}\right),
\end{aligned}
$$

where $(a, \alpha)$ are flat superspace indices, $M=(m, \mu)$ are curved indices and $\Upsilon$ is an arbitrary chiral superfield parameterizing the super-Weyl transformation. We will focus on a theory for $n+1$ chiral multiplets $\mathcal{Z}^{A}, A=0, \ldots, n$, that transform as follows under super-Weyl transformations:

$\mathcal{Z}^{A} \rightarrow \mathrm{e}^{-6 \Upsilon} \mathcal{Z}^{A}$

The chiral superfields $\mathcal{Z}^{A}$ comprise, in a democratic way, a super-Weyl compensator and $n$ physical multiplets.

The ordinary old minimal formulation of supergravity is obtained by choosing a super-Weyl compensator $Z$, e.g. $Z \equiv \mathcal{Z}^{0}$, and subject it to a gauge-fixing condition using the super-Weyl invariance. On the other hand, we will perform the duality transformation of the conventional chiral multiplets $\mathcal{Z}^{A}$ to three-form multiplets before gauge-fixing the super-Weyl invariance. In this way, the procedure will work exactly as in the rigid supersymmetry case, but will involve the super-Weyl compensator in addition to the physical chiral superfields. Gauge-fixing the super-Weyl symmetry afterwards will produce a Lagrangian describing the coupling of three-form multiplets to a supergravity multiplet with one or two gauge fields substituting the scalar auxiliary fields.

In the next section we will focus on pure supergravity and its three-form variants. The inclusion of additional physical chiral multiplets and a general superpotential of the form (1.1) will be considered in Sect. 3.2. The following discussion can include additional 'spectator' matter or gauge multiplets, which will not be explicitly indicated for notational simplicity.

\subsection{Variant minimal supergravities from duality}

We start by considering the minimal theory, in which the old minimal supergravity multiplet is coupled just to the superWeyl compensator $Z$, which transforms as in (3.3). Then, up to a complex constant rescaling of $Z$, the most general super-Weyl-invariant Lagrangian has the form

$$
\mathcal{L}=-3 \int \mathrm{d}^{4} \theta E(Z \bar{Z})^{\frac{1}{3}}+\left(c \int \mathrm{d}^{2} \Theta 2 \mathcal{E} Z+\text { c.c. }\right),
$$


in which $E$ denotes the Berezinian super-determinant of the super-vielbein, $\mathrm{d}^{2} \Theta 2 \mathcal{E}$ is a chiral superspace measure [45] and $c$ is an arbitrary complex number which gives rise to the gravitational cosmological constant and the gravitino mass. Under (3.2), the superspace measures rescale as

$E \rightarrow \mathrm{e}^{2(\Upsilon+\bar{\Upsilon})} E, \quad \mathrm{~d}^{2} \Theta \mathcal{E} \rightarrow \mathrm{e}^{6 \Upsilon} \mathrm{d}^{2} \Theta \mathcal{E}$.

Hence the super-Weyl invariance of the supergravity Lagrangian is manifest. We can now follow Sect. 2, distinguishing two cases.

\subsubsection{Single three-form supergravity}

We first proceed along the lines of Sect. 2.1, setting $c \equiv i r$, with real $r$, and promoting $r$ to a chiral multiplet $X$ by adding an appropriate Lagrange multiplier. ${ }^{6}$ Consider the modified Lagrangian

$$
\begin{aligned}
\mathcal{L}^{\prime}= & -3 \int \mathrm{d}^{4} \theta E(Z \bar{Z})^{\frac{1}{3}} \\
& +\left(\int \mathrm{d}^{2} \Theta 2 \mathcal{E}\left[X Z+\frac{1}{8}\left(\overline{\mathcal{D}}^{2}-8 \mathcal{R}\right)\{U(X+\bar{X})\}\right]+\text { c.c. }\right),
\end{aligned}
$$

where $U$ is a scalar real superfield and $\mathcal{R}$ is the chiral superfield curvature whose leading component is the auxiliary field $M=-\frac{1}{6} \mathcal{R} \mid$ of the gravity multiplet. Notice that (3.6) is super-Weyl invariant if we impose the requirement that

$U \rightarrow \mathrm{e}^{-2(\Upsilon+\bar{\Upsilon})} U$

under super-Weyl transformations, since $\overline{\mathcal{D}}^{2}-8 \mathcal{R} \rightarrow$ $\mathrm{e}^{-4 \Upsilon}\left(\overline{\mathcal{D}}^{2}-8 \mathcal{R}\right) \mathrm{e}^{2 \bar{\Upsilon}}$.

Integrating $U$ out of (3.6) by imposing its equation of motion implies that $X$ must be an arbitrary real constant $r$ and then one goes back to (3.4). Instead, integrating out $X$ gives

$Z \equiv Y$,

where the chiral superfield

$Y \equiv-\frac{1}{4}\left(\overline{\mathcal{D}}^{2}-8 \mathcal{R}\right) U$

is the natural generalization of the rigid single three-form multiplets discussed in Sect. 2.1. In particular, the bosonic three-form $A_{3}$ is contained in the component

\footnotetext{
${ }^{6}$ We choose a purely imaginary $c$ in order to obtain the single threeform supergravity in its most common form, as used for instance in $[8,13-15,32,33,35]$. Clearly, by a simple redefinition $Z \rightarrow-i Z$ one can make $c$ purely real.
}

$-\frac{1}{8} \bar{\sigma}_{m}^{\dot{\alpha} \alpha}\left[\mathcal{D}_{\alpha}, \overline{\mathcal{D}}_{\dot{\alpha}}\right] U \mid \equiv\left({ }^{*} A_{3}\right)_{m}$,

of $U$. The bosonic gauge transformation $A_{3} \rightarrow A_{3}+\mathrm{d} \Lambda_{2}$ is contained in the superfield gauge transformation $U \rightarrow$ $U+L$, where $L$ is an arbitrary linear multiplet. This gauge invariance allows one to write the superfield $U$ in an appropriate WZ gauge $U \mid=0$, which we have already used in (3.10).

By integrating out $Z$ one gets the equation

$X=-\frac{1}{4}\left(\overline{\mathcal{D}}^{2}-8 \mathcal{R}\right)\left[Z^{-\frac{2}{3}} \bar{Z}^{\frac{1}{3}}\right]$,

and by plugging (3.8) and (3.11) back into (3.6) one obtains the dual Lagrangian

$\hat{\mathcal{L}}=-3 \int \mathrm{d}^{4} \theta E(Y \bar{Y})^{\frac{1}{3}}+\mathcal{L}_{\text {bd }}$,

where

$\mathcal{L}_{\text {bd }}=\frac{1}{8} \int \mathrm{d}^{2} \Theta 2 \mathcal{E}\left(\overline{\mathcal{D}}^{2}-8 \mathcal{R}\right)\{U(X-\bar{X})\}+$ c.c.

Note that $\mathcal{L}_{\mathrm{bd}}$ is indeed a total derivative. $Y$ transforms as $Z$ under super-Weyl transformations $\left(Y \rightarrow \mathrm{e}^{-6 \Upsilon} Y\right)$ and plays the role of the super-Weyl compensator.

It is well known that different off-shell formulations of four-dimensional $\mathcal{N}=1$ supergravity can be obtained from its superconformal version by choosing different compensator fields [47-49]. Here the use of $Y$ as a compensator in the super-Weyl-invariant formulation leads, as was shown in [35], to the three-form minimal supergravity [8,13$15,32,33]$, in which the imaginary part of the old minimal auxiliary field $M$ is substituted by the Hodge dual of a real field strength $F_{4}=\mathrm{d} A_{3}$.

In order to see this, we can use the super-Weyl symmetry to set

$Y=1$

By recalling the definition of $Y$ given in (3.9) and its expansion (2.8) and skipping the dependence on the fermions, the lowest component of this equation gives $Y \mid=1$ while the highest component $-\frac{1}{4} \mathcal{D}^{2} Y \mid=0$ gives $\operatorname{ImM}+{ }^{*} \mathrm{dA}_{3}=0$, so that the conventional scalar auxiliary field of the supergravity multiplet has the form $M=\operatorname{Re} M-i^{*} F_{4}$, as proposed in [32] and discussed in detail in [8]. Hence, the component fields of the supergravity multiplet of this formulation are

$e_{a}^{m}, \quad \psi_{m}^{\alpha}, \quad b_{a}, \quad M_{0}, \quad A_{3}$,

where $M_{0} \equiv \operatorname{Re} M$ is a real scalar. 


\subsubsection{Double three-form supergravity}

In order to arrive at the minimal double three-form supergravity [28] we must promote the entire arbitrary constant $c$ to a dynamical chiral field $X$ and proceed as in the previous examples. This can be done by starting from the Lagrangian

$$
\begin{aligned}
\mathcal{L}^{\prime}= & -3 \int \mathrm{d}^{4} \theta E(Z \bar{Z})^{\frac{1}{3}} \\
& +\left(\int \mathrm{d}^{2} \Theta 2 \mathcal{E}\left[X Z+\frac{1}{4}\left(\overline{\mathcal{D}}^{2}-8 \mathcal{R}\right)\{\bar{X} \Sigma\}\right]+\text { c.c. }\right),
\end{aligned}
$$

where $\Sigma=\overline{\mathcal{D}} \bar{\Psi}$ is a complex linear superfield, the locally supersymmetric generalization of the complex linear superfield introduced in Sect. 2.2. The components of $\Sigma$ in the appropriate $\mathrm{WZ}$ gauge are

$$
\begin{aligned}
\Sigma \mid & =0, \\
\mathcal{D}^{2} \Sigma \mid & =-4 \bar{s}, \\
\frac{1}{2} \bar{\sigma}_{m}^{\dot{\alpha} \alpha}\left[\mathcal{D}_{\alpha}, \overline{\mathcal{D}}_{\dot{\alpha}}\right] \Sigma \mid & =-i C_{m}, \\
\mathcal{D}^{2} \overline{\mathcal{D}}^{2} \bar{\Sigma} \mid & =8^{*} \bar{G}_{4}+16 \bar{M} s,
\end{aligned}
$$

with $G_{4} \equiv \mathrm{d} C_{3}$ and $C_{m} \equiv\left({ }^{*} C_{3}\right)_{m}$. One can go to this gauge because of the invariance of the construction under the superfield gauge transformation of the form (2.29)-(2.30).

The action (3.16) is invariant under super-Weyl transformations if $\Psi_{\alpha}$, and eventually $\Sigma$, transform as follows [48]:

$\Psi_{\alpha} \rightarrow \mathrm{e}^{-3 \Upsilon} \Psi_{\alpha}, \quad \Sigma \rightarrow \mathrm{e}^{-2(\Upsilon+\bar{\Upsilon})} \Sigma$.

As in the previous examples, by integrating out $\Psi_{\alpha}$ one gets back (3.4). On the other hand, by integrating out $X$ and $Z$ one finds

$Z=S \equiv-\frac{1}{4}\left(\overline{\mathcal{D}}^{2}-8 \mathcal{R}\right) \bar{\Sigma}$,

$X=-\frac{1}{4}\left(\overline{\mathcal{D}}^{2}-8 \mathcal{R}\right)\left[Z^{-\frac{2}{3}} \bar{Z}^{\frac{1}{3}}\right]$.

After inserting these expressions into the Lagrangian one arrives at the dual description

$$
\begin{aligned}
\hat{\mathcal{L}}= & -3 \int \mathrm{d}^{4} \theta E(S \bar{S})^{\frac{1}{3}} \\
& +\frac{1}{4}\left[\int \mathrm{d}^{2} \Theta 2 \mathcal{E}\left(\overline{\mathcal{D}}^{2}-8 \mathcal{R}\right)\{\bar{X} \Sigma-X \bar{\Sigma}\}+\text { c.c. }\right],
\end{aligned}
$$

where $S$ is a double three-form multiplet which plays the role of the super-Weyl compensator. Note that $X$ and $S$ in (3.20) are given by (3.19), and that the second term in (3.20) is the boundary term.
One can then gauge-fix the super-Weyl invariance by putting $S=1$ and find that

$M=-\frac{1}{2} * G_{4}$.

Hence the supergravity multiplet in this formulation becomes

$e_{a}^{m}, \quad \psi_{m}^{\alpha}, \quad b_{m}, \quad C_{3}$,

where $C_{3}$ is a complex three-form. Therefore we refer to this formulation as double three-form supergravity. The bosonic sector of this minimal supergravity theory follows from the Lagrangian (3.20) and has the following form:

$\mathrm{e}^{-1} \hat{\mathcal{L}}=-\frac{1}{2} R+\frac{1}{3} b^{m} b_{m}-\frac{1}{12}\left|{ }^{*} G_{4}\right|^{2}+\frac{1}{12} \mathcal{D}_{m}\left(C^{m}{ }^{*} \bar{G}_{4}+\right.$ c.c. $)$.

The equations of motion of $C_{3}$ have general solution

${ }^{*} G_{4}=6 c$.

If we integrate out $C_{3}$ by inserting (3.24) into the Lagrangian (3.23) we find the standard supergravity theory with a negative cosmological constant. Notice that (3.23) has a welldefined variation with respect to $C_{3}$ thanks to the presence of the boundary term. As in the previous sections, this is guaranteed by our duality procedure once one appropriately chooses the form of the Lagrange multiplier term in (3.16).

\subsection{Three-form matter-coupled supergravities}

In the previous section we obtained known minimal threeform supergravities with the use of the locally supersymmetric counterpart of the duality procedure described in Sect. 2 . We now pass to the considerably more general case outlined at the beginning of this section. We consider a super-Weylinvariant supergravity theory coupled to $n+1$ chiral superfields $\mathcal{Z}^{A}$ which transform as in (3.3). We stress once again that, even if not explicitly indicated for notational simplicity, additional spectator chiral and vector multiplets may be included without difficulties (as in the example discussed in Sect. 4). is

The general form of the super-Weyl-invariant Lagrangian

$\mathcal{L}=-3 \int \mathrm{d}^{4} \theta E \Omega(\mathcal{Z}, \overline{\mathcal{Z}})+\int \mathrm{d}^{2} \Theta 2 \mathcal{E} \mathcal{W}(\mathcal{Z})$,

where the kinetic potential $\Omega(\mathcal{Z}, \overline{\mathcal{Z}})$ and the superpotential $\mathcal{W}(\mathcal{Z})$ have the following homogeneity properties:

$\Omega(\lambda \mathcal{Z}, \bar{\lambda} \overline{\mathcal{Z}})=|\lambda|^{\frac{2}{3}} \Omega(\mathcal{Z}, \overline{\mathcal{Z}}), \quad \mathcal{W}(\lambda \mathcal{Z})=\lambda \mathcal{W}(\mathcal{Z})$. 
Before discussing the duality procedure, let us briefly recall how this formulation is related to the more standard supergravity formulation. First, one singles out a super-Weyl compensator $Z$ as follows:

$\mathcal{Z}^{A}=Z \mathcal{Z}_{0}^{A}(\Phi)$

where $\mathcal{Z}_{0}^{A}(\Phi)$ is a set of functions of the physical chiral multiplets $\Phi^{i}(i=1, \ldots, n)$, which are inert under the superWeyl transformations. Clearly, the split (3.27) has a large arbitrariness and one may redefine

$Z \rightarrow \mathrm{e}^{-f(\Phi)} Z, \quad \mathcal{Z}_{0}^{A}(\Phi) \rightarrow \mathrm{e}^{f(\Phi)} \mathcal{Z}_{0}^{A}(\Phi)$.

The kinetic potential $\Omega(\mathcal{Z}, \overline{\mathcal{Z}})$ can be written as follows:

$\Omega(\mathcal{Z}, \overline{\mathcal{Z}})=|Z|^{\frac{2}{3}} \mathrm{e}^{-\frac{1}{3} K(\Phi, \bar{\Phi})}$,

where $K(\Phi, \bar{\Phi}) \equiv-3 \log \Omega\left(\mathcal{Z}_{0}(\Phi), \overline{\mathcal{Z}}_{0}(\bar{\Phi})\right)$ is the ordinary Kähler potential. Note that the possibility of making the redefinition (3.28) corresponds to the invariance under Kähler transformations $K(\Phi, \bar{\Phi}) \rightarrow K(\Phi, \bar{\Phi})-f(\Phi)-\bar{f}(\bar{\Phi})$. The conventional superpotential $W(\Phi)$ is singled out by using the split (3.27) and defining

$\mathcal{W}(\mathcal{Z})=Z W(\Phi)$

where $W(\Phi) \equiv \mathcal{W}\left(\mathcal{Z}_{0}(\Phi)\right)$. Under the redefinition (3.28) $W$ transforms as follows: $W(\Phi) \rightarrow \mathrm{e}^{f(\Phi)} W(\Phi)$. The conventional formulation can then be obtained by gauge-fixing the super-Weyl invariance, e.g. by putting

$Z=1$.

In order to perform the duality procedure, let us come back to the super-Weyl-invariant Lagrangian (3.25) and consider the superpotential of the form

$\mathcal{W}(\mathcal{Z}) \equiv e_{A} \mathcal{Z}^{A}+m^{B} \mathcal{G}_{B A}(\mathcal{Z}) \mathcal{Z}^{A}+\hat{\mathcal{W}}(\mathcal{Z})$.

The homogeneity condition (3.26) requires that $\mathcal{G}_{A B}(\lambda \mathcal{Z})=$ $\mathcal{G}_{A B}(\mathcal{Z})$ and $\hat{\mathcal{W}}(\lambda \mathcal{Z})=\lambda \hat{\mathcal{W}}(\mathcal{Z})$. Though the construction under consideration can be applied to generic $\mathcal{G}_{A B}$, we will restrict ourselves to the case in which

$\mathcal{G}_{A B}(\mathcal{Z}) \equiv \partial_{A} \partial_{B} \mathcal{G}(\mathcal{Z})$

with $\mathcal{G}(\mathcal{Z})$ being a (possibly locally defined) homogeneous prepotential of degree $2 \mathcal{G}(\lambda \mathcal{Z})=\lambda^{2} \mathcal{G}(\mathcal{Z})$ defining a local special Kähler space parametrized by homogeneous coordinates $\mathcal{Z}^{A}, A=0,1, \ldots, n .^{7}$ As we will see, string flux com-

\footnotetext{
7 The minimal supergravities considered in Sect. 3.1 correspond to the simplest subcases with $n=0, \mathcal{Z}^{0}=Z$ and $c \equiv e_{0}+\mathcal{G}_{00} m^{0}$, where $\mathcal{G}_{00}$
}

pactifications have superpotentials of this kind with $\left(e_{A}, m^{B}\right)$ representing appropriately quantized units of fluxes.

We would like to make the $2 n+2$ constants $\left(e_{A}, m^{A}\right)$ in (3.32) dynamical, i.e. to replace them with the field strengths of $2 n+2$ three-forms. This is achieved by dualizing the chiral fields $\mathcal{Z}^{A}$, easily adapting the procedure introduced in Sect. 2.3 for the rigid supersymmetric case. As in that section, we assume that $\mathcal{M}_{A B}$ defined as in (2.38) is invertible. (The case of degenerate $\mathcal{M}_{A B}$ can be addressed as outlined in Sect. 2, combining dualizations to single and double threeform multiplets.) First, we substitute the chiral superspace integral of the superpotential term (3.32) with

$$
\begin{aligned}
\mathcal{L}_{X}= & 2 \int \mathrm{d}^{2} \Theta \mathcal{E}\left(X_{A} \mathcal{Z}^{A}-\frac{1}{4}\left(\overline{\mathcal{D}}^{2}-8 \mathcal{R}\right)\right. \\
& \left.\times\left[\mathcal{M}^{A B}\left(X_{A}-\bar{X}_{A}\right) \Sigma_{B}\right]+\hat{\mathcal{W}}(\mathcal{Z})\right),
\end{aligned}
$$

where, as in the rigid supersymmetry case, $\mathcal{M}^{A B}$ is inverse of $\mathcal{M}_{A B}=\operatorname{Im} \mathcal{G}_{A B}, X_{A}$ are chiral superfields and $\Sigma_{A}$ are complex linear superfields $\Sigma_{A} \equiv \overline{\mathcal{D}}_{\dot{\alpha}} \bar{\Psi}_{A}^{\dot{\alpha}}$. Upon integrating out $\Psi_{A}^{\alpha}$ one gets back (3.25). On the other hand, by integrating out $X_{A}$ and $\mathcal{Z}^{A}$ one finds

$\mathcal{Z}^{A}=S^{A}$

where the chiral superfields $S^{A}$ are double three-form multiplets, defined by the generalization of (2.44),

$$
\begin{aligned}
S^{A}= & \frac{1}{4}\left(\overline{\mathcal{D}}^{2}-8 \mathcal{R}\right)\left[\mathcal{M}^{A B}\left(\Sigma_{B}-\bar{\Sigma}_{B}\right)\right], \\
X_{A}= & -\hat{\mathcal{W}}_{A}+\frac{1}{4}\left(\overline{\mathcal{D}}^{2}-8 \mathcal{R}\right) \\
& \times\left[\Omega_{A}+\frac{\partial \mathcal{M}^{B C}}{\partial S^{A}}\left(X_{B}-\bar{X}_{B}\right)\left(\Sigma_{C}-\bar{\Sigma}_{C}\right)\right] .
\end{aligned}
$$

The Lagrangian then reads

$$
\hat{\mathcal{L}}=-3 \int \mathrm{d}^{4} \theta E \Omega(S, \bar{S})+\left(\int \mathrm{d}^{2} \Theta 2 \mathcal{E} \hat{\mathcal{W}}(S)+\text { c.c. }\right)+\mathcal{L}_{\text {bd }},
$$

in which the boundary term is given by the $X$-dependent part of (3.34) once one replaces $\mathcal{Z}^{A}$ with $S^{A}$ and $X_{A}$ with (3.37). Note that, as in the rigid supersymmetry case, the dual Lagrangian does not have the part of the superpotential that depended on $e_{A}$ and $m^{A}$. We thus end up with a theory in which the only independent superfields are the complex linear multiplets $\Sigma_{A}$.

Footnote 7 continued

is necessarily constant by homogeneity. In particular, the single threeform minimal supergravity of Sect. 3.1.1 is obtained by setting $\mathcal{G}_{00}=0$ and redefining $Z \rightarrow i Z$. 
The double three-form multiplets $S^{A}$ are defined by (3.36), in which $\mathcal{M}^{A B}$ should be considered as a function of $S^{A}$ and $\bar{S}^{A}$. Hence (3.36) is non-linear and so is not generically solvable for $S^{A}$ as functions of $\Sigma_{A}$. However, it turns out to be tractable for superfield components. For simplicity, we will restrict ourselves to the bosonic ones setting the fermionic components equal to zero. Using the local symmetry (2.45) we can impose the Wess-Zumino gauge in which, in particular, $\Sigma_{A} \mid=0$. Then the remaining bosonic components of $\Sigma_{A}$ are

$$
\begin{aligned}
\mathcal{D}^{2} \Sigma_{A} \mid & =-4 \bar{s}_{A}, \\
\frac{1}{2} \bar{\sigma}_{m}^{\dot{\alpha} \alpha}\left[\mathcal{D}_{\alpha}, \overline{\mathcal{D}}_{\dot{\alpha}}\right] \Sigma_{A} \mid & =-\tilde{A}_{A m}-\mathcal{G}_{A B} A_{m}^{B}, \\
\mathcal{D}^{2} \overline{\mathcal{D}}^{2} \bar{\Sigma}_{A} \mid & =8 i \mathcal{D}_{m}\left(\tilde{A}_{A}^{m}+\overline{\mathcal{G}}_{A B} A^{B m}\right)+16 \bar{M} s_{A},
\end{aligned}
$$

where $A_{m}^{A} \equiv\left({ }^{*} A_{3}^{A}\right)_{m}$ and $\tilde{A}_{A m} \equiv\left({ }^{*} \tilde{A}_{3 A}\right)_{m}$.

From (3.36) it follows that the scalar component $s_{A}$, with lower indices, appearing in (3.39) is related to $s^{A} \equiv S^{A} \mid$, with upper indices, by the inverse metric $\mathcal{M}^{A B}$. Since $S^{A} \mid \equiv$ $\mathcal{Z}^{A} \mid \equiv z^{A}$, we can use $z^{A}$ instead of $s^{A}$ and write this relation as follows:

$z^{A}=\mathcal{M}^{A B}(z, \bar{z}) s_{B}$.

In general it is not possible to explicitly invert the above expression and express $z^{A}$ in terms of the scalar fields $s_{A}$ of the complex linear superfield $\bar{\Sigma}_{A}$. Hence, in what follows it will be more convenient to use $z^{A}$ as independent scalar fields in the component Lagrangians which we will shortly present. The $\theta^{2}$-component of (3.36) is then

$$
\begin{aligned}
& F_{S}^{A} \equiv-\frac{1}{4} \mathcal{D}^{2} S^{A} \mid=\bar{M} z^{A}+\frac{i}{2} \mathcal{M}^{A B} \\
& \quad \times\left({ }^{*} \tilde{F}_{4 B}+\overline{\mathcal{G}}_{B C}{ }^{*} F_{4}^{C}+2 \operatorname{Re}\left[\overline{\mathcal{G}}_{B C D} \bar{F}_{S}^{D} \bar{z}^{C}\right]\right),
\end{aligned}
$$

where $\tilde{F}_{4 A}=\mathrm{d} \tilde{A}_{3 A}, F_{4}^{A}=\mathrm{d} A_{3}^{A}$ and $\mathcal{G}_{A B C} \equiv \partial_{A} \mathcal{G}_{B C}$. Now, taking into account that $z^{A} \mathcal{G}_{A B C}=0$ by homogeneity, we reduce Eq. (3.41) to

$F_{S}^{A}=\bar{M} z^{A}+\frac{i}{2} \mathcal{M}^{A B}\left({ }^{*} \tilde{F}_{4 B}+\overline{\mathcal{G}}_{B C}{ }^{*} F_{4}^{C}\right)$.

To fix the super-Weyl invariance it turns out to be convenient to choose one of the superfields $S^{A}(A=(0, i))$, say $S^{0}$, as the super-Weyl compensator and impose

$S^{0}=1$.

The superspace condition (3.43) implies the component field conditions $z^{0}=1$ and $F_{S}^{0}=0$. The bosonic relations (3.42) split as follows:

$$
\begin{aligned}
\bar{M} & =-\frac{i}{2} \mathcal{M}^{0 B}(z, \bar{z})\left[{ }^{*} \tilde{F}_{4 B}+\overline{\mathcal{G}}_{B C}(\bar{z})^{*} F_{4}^{C}\right], \\
F_{S}^{i} & =\bar{M} z^{i}+\frac{i}{2} \mathcal{M}^{i B}(z, \bar{z})\left[{ }^{*} \tilde{F}_{4 B}+\overline{\mathcal{G}}_{B C}(\bar{z})^{*} F_{4}^{C}\right],
\end{aligned}
$$

where $z^{i} \equiv S^{i} \mid$ and $F_{S}^{i} \equiv-\frac{1}{4} \mathcal{D}^{2} S^{i} \mid$ are the lowest and highest scalar components of the three-form multiplets $S^{i}$ $(i=1, \ldots, n)$.

After having gauge-fixed the super-Weyl symmetry, the Lagrangian describing the coupling of the $S^{i}$ superfields to supergravity takes the form

$$
\begin{aligned}
\hat{\mathcal{L}}= & -3 \int \mathrm{d}^{4} \theta E \mathrm{e}^{-3 K(S, \bar{S})} \\
& +\left(\int \mathrm{d}^{2} \Theta 2 \mathcal{E} \hat{W}(S)+\text { c.c. }\right)+\mathcal{L}_{\text {bd }} .
\end{aligned}
$$

Note that in this Lagrangian the scalar auxiliary fields of the gravity and matter multiplets are defined by (3.44) (ignoring fermions).

\section{Application to type IIA flux compactifications}

As an application of the above dualization procedure, we will now consider an example of type IIA flux compactifications of string theory on a Calabi-Yau three-fold $\mathrm{CY}_{3}$ in the presence of O6-planes. In particular, we will focus on the effective theory obtained by turning on RR fluxes in the internal $\mathrm{CY}_{3}$ space. For simplicity, we will also set the internal NSNS flux $\mathrm{H}_{3}$ to zero, so that the tadpole condition just requires that the O6 charge is canceled by the presence of D6-planes, without involving the RR fluxes.

We will focus on the closed string scalar spectrum. The relevant terms in the effective $\mathcal{N}=1$ supergravity for these kinds of compactifications can be found in [50]. The closed string moduli $v^{i}(x)$ and $b^{i}(x), i=1, \ldots, h_{-}^{1,1}\left(C Y_{3}\right)$, are obtained by expanding the Kähler form $J$ and the NSNS two-form $B_{2}$ in a basis of orientifold-odd integral harmonic 2-forms $\omega_{i} \in H_{-}^{2}(X ; \mathbb{Z})$

$J=v^{i} \omega_{i}, \quad B_{2}=b^{i} \omega_{i}$.

These moduli, together with their supersymmetric partners, combine into $n \equiv h_{-}^{1,1}\left(\mathrm{CY}_{3}\right)$ chiral superfields $\Phi^{i}$ with lowest components

$\Phi^{i} \mid=\varphi^{i}=v^{i}-i b^{i}$.

Furthermore, the complex structure, the dilaton, the internal RR three-form moduli and the associated supersymmetric partners combine into additional chiral superfields $T^{q}, q=$ 
$1, \ldots, h^{2,1}\left(C Y_{3}\right)+1$. The effective supergravity theory is characterised by the following Kähler potential ${ }^{8}$ :

$\mathcal{K}(\Phi, \bar{\Phi}, T, \bar{T})=K(\Phi, \bar{\Phi})+\hat{K}(T, \bar{T})$.

In the following we will not need the explicit form of $\hat{K}(T, \bar{T})$, but we will just use the fact that it satisfies the condition

$\hat{K}^{\bar{r} q} \hat{K}_{q} \hat{K}_{\bar{r}}=4$

where $\hat{K}_{q} \equiv \frac{\partial \hat{K}}{\partial T^{q}}, \hat{K}_{q \bar{r}} \equiv \frac{\partial^{2} \hat{K}}{\partial T^{q} \partial \bar{T}^{\bar{q}}}, \ldots$, and $\hat{K}^{\bar{r} q}$ is the inverse of the Kähler metric $\hat{K}_{q \bar{r}}$. Similarly, $K_{i} \equiv \frac{\partial K}{\partial \Phi^{i}}$, $K_{i \bar{\jmath}} \equiv \frac{\partial^{2} K}{\partial \Phi^{i} \partial \bar{\Phi}^{\bar{j}}}, \ldots$, and $K^{\bar{j} i}$ is the inverse of the Kähler metric $K_{i \bar{\jmath}}$.

The Kähler potential $K(\Phi, \bar{\Phi})$ is given by

$K(\Phi, \bar{\Phi})=-\log \left[\frac{1}{3 !} k_{i j k}\left(\operatorname{Re} \Phi^{i}\right)\left(\operatorname{Re} \Phi^{j}\right)\left(\operatorname{Re} \Phi^{k}\right)\right]$

where $k_{i j k}$ are the intersection numbers

$k_{i j k}=\int_{\mathrm{CY}_{3}} \omega_{i} \wedge \omega_{j} \wedge \omega_{k}$

Notice that $K(\Phi, \bar{\Phi})$ depends only on the real combinations $\Phi^{i}+\bar{\Phi}^{i}$, so that we can make the identification $K_{i} \equiv K_{\bar{l}}$. We will also use the fact that $K(\Phi, \bar{\Phi})$ satisfies the no-scale condition

$K^{\bar{j} i} K_{i} K_{\bar{\jmath}}-3=0$.

The flux-induced superpotential is of the form introduced in [51-53] and depends only on the chiral superfields $\Phi^{i} 9$

$W=e_{0}+i e_{i} \Phi^{i}-\frac{1}{2} k_{i j k} m^{i} \Phi^{j} \Phi^{k}+\frac{i}{6} m^{0} k_{i j k} \Phi^{i} \Phi^{j} \Phi^{k}$,

where $e_{0}, e_{i}, m^{i}$ and $m^{0}$ represent the flux quanta of the internal RR fields.

\subsection{Dualization to the three-form effective theory}

The effective theory described above has exactly the same structure as the theories considered in Sect. 3.2, up to the explicit presence of a spectator sector given by the chiral fields $T^{r}$. In order to make this similarity manifest, we rewrite

$\overline{8}$ The formulas of [50] are valid in the large volume and constant warping approximation, which then neglects the back-reaction of the fluxes and branes on the underlying Calabi-Yau geometry. The back-reaction of fluxes and branes is expected to break the split structure of (4.3).

${ }^{9}$ In what follows we will tend to use notation close to that of $[25,44]$. this theory in a super-Weyl-invariant form by adding a superWeyl compensator $Z$ and combining it with the chiral fields $\Phi^{i}$ into $n+1$ chiral superfields $\mathcal{Z}^{A}=\left(\mathcal{Z}^{0}, \mathcal{Z}^{i}\right)$ such that

$\mathcal{Z}^{0} \equiv Z$

and

$\mathcal{Z}^{i} \equiv i Z \Phi^{i}$

which transform as in (3.3) under the super-Weyl transformations. Then it is easy to see that the superpotential (4.8) gets transformed into (3.30) of the form

$\mathcal{W}(\mathcal{Z})=e_{A} \mathcal{Z}^{A}+\frac{1}{2 \mathcal{Z}^{0}} m^{i} k_{i j k} \mathcal{Z}^{j} \mathcal{Z}^{k}-\frac{1}{6\left(\mathcal{Z}^{0}\right)^{2}} m^{0} k_{i j k} \mathcal{Z}^{i} \mathcal{Z}^{j} \mathcal{Z}^{k}$.

This clearly satisfies the homogeneity condition (3.26) and can be written in the form (3.32) with $\hat{\mathcal{W}}(\mathcal{Z})=0$ and $\mathcal{G}_{A B}=$ $\partial_{A} \partial_{B} \mathcal{G}(\mathcal{Z})$, where

$\mathcal{G}(\mathcal{Z})=\frac{1}{6 \mathcal{Z}^{0}} k_{i j k} \mathcal{Z}^{i} \mathcal{Z}^{j} \mathcal{Z}^{k}$

We are now in a position to apply the duality transformation described in Sect. 3.2. After dualization and gauge-fixing the super-Weyl symmetry by setting

$Z=S^{0}=1$

the final result is a Lagrangian of the form (3.45) with $\hat{W}=0$ and a Kähler potential which is modified by a contribution of the 'spectators' $T^{r}$

$\mathcal{L}=-3 \int \mathrm{d}^{4} \theta E \mathrm{e}^{-3 K(S, \bar{S})-3 \hat{K}(T, \bar{T})}+\mathcal{L}_{\text {bd }}$

Moreover, the superpotential has completely disappeared from the dual effective theory, since it is now encoded in the structure of the constrained superfields (3.36).

Notice that because of the definition (4.10), after dualization and gauge-fixing we have $\Phi^{i}=-i S^{i}$ and we can identify the lowest components as follows:

$S^{i} \mid \equiv z^{i}=i \varphi^{i}$

In the following it will also be convenient to use

$F^{i} \equiv-i F_{S}^{i}$

instead of $F_{S}^{i}$, such that $-\frac{1}{4} \mathcal{D}^{2} \Phi^{i}=F^{i}$.

Upon setting to zero the fermions, the independent bosonic components of these superfields are given by (3.40) and 
(3.44). The latter take the following form in the case under consideration $^{10}$

$\operatorname{Re} M=\frac{1}{2} * \mathcal{F}_{4}^{0}$,

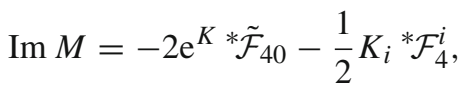

$\operatorname{Re} F^{i}=\frac{1}{4} * \mathcal{F}_{4}^{0} v^{i}-\mathrm{e}^{K}\left(K^{i j}-2 v^{i} v^{j}\right) * \tilde{\mathcal{F}}_{4 j}$,

$\operatorname{Im} F^{i}=2 \mathrm{e}^{K *} \tilde{\mathcal{F}}_{40} v^{i}+\frac{1}{2}\left({ }^{*} \mathcal{F}_{4}^{i}+v^{i} K_{j}{ }^{*} \mathcal{F}_{4}^{j}\right)$,

where the four-forms $\mathcal{F}_{4}^{A}$ and $\tilde{\mathcal{F}}_{4 A}$ are defined in terms of the field strengths $F_{4}^{A}=\mathrm{d} A_{3}^{A}$ and $\tilde{F}_{4 A}=\mathrm{d} \tilde{A}_{3 A}$ as follows:

$\mathcal{F}_{4}^{0}=-F_{4}^{0}, \quad \mathcal{F}_{4}^{i}=-F_{4}^{i}+b^{i} F_{4}^{0}$,

$\tilde{\mathcal{F}}_{4 i}=\tilde{F}_{4 i}+k_{i j k} b^{j} F_{4}^{k}-\frac{1}{2} k_{i j k} b^{j} b^{k} F_{4}^{0}$,

$\tilde{\mathcal{F}}_{40}=\tilde{F}_{40}+b^{i} \tilde{F}_{4 i}+\frac{1}{2} k_{i j k} b^{i} b^{j} F_{4}^{k}-\frac{1}{6} k_{i j k} b^{i} b^{j} b^{k} F_{4}^{0}$.

Note that the four-forms $\mathcal{F}_{4}^{A}$ and $\tilde{\mathcal{F}}_{4 A}$ have exactly the same structure as the four-forms obtained in $[25,44]$ upon dimensional reduction of the type IIA RR field strengths, ${ }^{11}$ which is quite encouraging. To convince ourselves that this is not a mere coincidence, in the following section we will compute the on-shell values of (4.18) by solving the equations of motion of $A_{3}^{A}$ and $\tilde{A}_{3 A}$, which follow from the dual Lagrangian (4.14). As we will see, these on-shell values perfectly match those obtained by ten-to-four-dimensional reduction $[25,44]$.

The bosonic part of the dual Lagrangian (4.14) can be computed by setting to zero fermionic component fields, integrating over the Grassmann variables and integrating out the supergravity auxiliary vector field. Finally, one can go to the Einstein frame by performing a Weyl rescaling of the vielbeins, the dual four-form field strengths and the component fields in (4.17) as follows:

$$
\begin{aligned}
\mathrm{e} a_{m} & \rightarrow \mathrm{e} a_{m} \mathrm{e} \frac{1}{6}(K+\hat{K}), \quad M \rightarrow M \mathrm{e}-\frac{2}{3}(K+\hat{K}), \\
F^{i} & \rightarrow F^{i} \mathrm{e}-\frac{2}{3}(K+\hat{K}), \quad F_{T}^{q} \rightarrow F_{T}^{q} \mathrm{e}-\frac{2}{3}(K+\hat{K}) .
\end{aligned}
$$

The result is the following bosonic Lagrangian:

$$
\begin{aligned}
\mathrm{e}^{-1} \mathcal{L}_{\text {bos }}= & -\frac{1}{2} R-K_{i \bar{j}}(\varphi, \bar{\varphi}) \partial \varphi^{i} \partial \bar{\varphi}^{\bar{\jmath}}-\hat{K}_{q \bar{r}}(t, \bar{t}) \partial t^{q} \partial \overline{t^{r}} \\
& +\mathrm{e}^{-1} \mathcal{L}_{3 \text {-form }},
\end{aligned}
$$

\footnotetext{
${ }_{10}$ To arrive at these relations we have used the specific form of the Kähler and superpotenional associated to the type IIA compactification in question given in Appendix C.

11 The structure of these field strengths reflects the $B_{2}$-twisting of the ten-dimensional RR-fields in the so called A-basis of the democratic formulation of [54], which provides a duality-symmetric description of the type IIA supergravity theory, whose $E_{11}$ origin was revealed in $[55,56]$.
}

in which $t^{q} \equiv T^{q} \mid$ and $\mathcal{L}_{3 \text {-form }}$ contains the three-form sector encoded in $M$ and $F^{i}$ as in (4.17) and the auxiliary fields $F_{T}^{q}$ of the $T^{q}$ multiplets

$$
\begin{aligned}
\mathrm{e}^{-1} \mathcal{L}_{3 \text {-form }}= & \mathrm{e}^{-\mathcal{K}} K_{i \bar{j}} F^{i} \bar{F}^{\bar{j}}+\mathrm{e}^{-\mathcal{K}} \hat{K}_{q \bar{r}} F_{T}^{q} \bar{F}_{T}^{\bar{r}} \\
& -\frac{1}{3} \mathrm{e}^{-\mathcal{K}}\left(M+K_{\bar{\imath}} \bar{F}^{\bar{\imath}}+\hat{K}_{\bar{q}} \bar{F}_{T}^{\bar{q}}\right) \\
& \times\left(\bar{M}+K_{i} F^{i}+\hat{K}_{q} F_{T}^{q}\right)+\mathcal{L}_{\text {bd }} .
\end{aligned}
$$

where we recall that $\mathcal{K} \equiv K+\hat{K}$. With the help of the no-scale condition (4.4), we can easily integrate out the auxiliary fields $F_{T}^{q}$ by solving their equations of motion, whose solution is

$\hat{K}_{q \bar{r}} \bar{F}_{T}^{\bar{r}}=-\left(M+K_{\bar{l}} \bar{F}^{\bar{\imath}}\right) \hat{K}_{q}$.

Substituting it back into the Lagrangian (4.21) and using (4.17) we obtain the following Lagrangian for the gauge three-forms:

$$
\begin{gathered}
\mathrm{e}^{\hat{K}} \mathrm{e}^{-1} \mathcal{L}_{3 \text {-form }}=\frac{\mathrm{e}^{-K}}{16}\left(* \mathcal{F}_{4}^{0}\right)^{2}+\mathrm{e}^{K} K^{i j *} \tilde{\mathcal{F}}_{4 i} * \tilde{\mathcal{F}}_{4 j} \\
+\frac{\mathrm{e}^{-K}}{4} K_{i j} * \mathcal{F}_{4}^{i *} \mathcal{F}_{4}^{j}+4 \mathrm{e}^{K}\left(* \tilde{\mathcal{F}}_{40}\right)^{2}+\mathcal{L}_{\text {bd }},
\end{gathered}
$$

with the boundary term

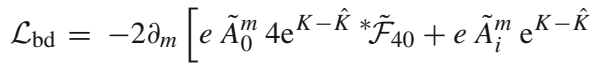

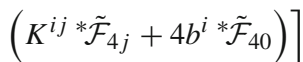

$$
\begin{aligned}
& +2 \partial_{m}\left[e A ^ { m i } \mathrm { e } ^ { - \hat { K } } \left(\frac{1}{4} \mathrm{e}^{-K} K_{i j}{ }^{*} \mathcal{F}_{4}^{j}-k_{i j k} b^{j} \mathrm{e}^{K} K^{k l} * \tilde{\mathcal{F}}_{4 l}\right.\right. \\
& \left.-2 k_{i j k} b^{j} b^{\left.k * \tilde{\mathcal{F}}_{40}\right)}\right]
\end{aligned}
$$

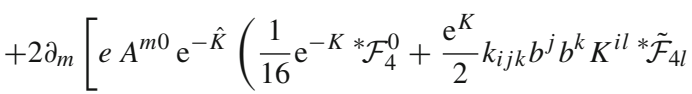

$$
\begin{aligned}
& \left.\left.-\frac{\mathrm{e}^{-K}}{4} b^{i} K_{i j}{ }^{*} \mathcal{F}_{4}^{j}+\frac{2}{3} k_{i j k} b^{i} b^{j} b^{k} \mathrm{e}^{K} *_{\mathcal{F}_{40}}\right)\right],
\end{aligned}
$$

where we recall that $A_{m}^{A} \equiv\left({ }^{*} A_{3}^{A}\right)_{m}$ and $\tilde{A}_{A m} \equiv\left({ }^{*} \tilde{A}_{3 A}\right)_{m}$. This boundary term is directly extracted by writing the superspace Lagrangian (3.34) in components.

The Lagrangian (4.20)-(4.24) provides a non-trivial example of the effect of the non-linear dualization procedure put forward in this paper. We explicitly see that it does not depend on the constants $e_{A}$ and $m^{A}$ appearing in the original Lagrangian and does not contain any potential for the scalar fields. Rather, as we will discuss in the next section, it is generated dynamically by the gauge three-forms $A_{3}^{A}$ and $\tilde{A}_{3 A}$.

\subsection{Back to the original theory}

Let us show how the bosonic Lagrangian of the original theory is reproduced from the bosonic Lagrangian (4.20). This is 
done by integrating out the gauge three-forms $A_{3}^{A}$ and $\tilde{A}_{3 A}$, which enter $\mathcal{F}_{4}^{A}$ and $\tilde{\mathcal{F}}_{4 A}$ as defined in (4.18). Indeed, the integration of the equations of motion which follow from (4.23) produces the following expressions involving $2 n+2$ integration constants which, for obvious reasons, we call $e_{A}$ and $m^{A}$ :

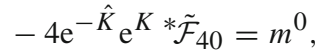

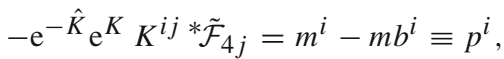

$$
\begin{aligned}
& -\frac{1}{4} \mathrm{e}^{-(K+\hat{K})} K_{i j}{ }^{*} \mathcal{F}_{4}^{j}=e_{i}+k_{i j k} b^{j} m^{k}-\frac{1}{2} k_{i j k} b^{j} b^{k} m^{0} \equiv \rho_{i}, \\
& -\frac{1}{16} \mathrm{e}^{-(K+\hat{K}) * \mathcal{F}_{4}^{0}=e_{0}+b^{i} e_{i}+\frac{1}{2} k_{i j k} b^{i} b^{j} m^{k}} \\
& -\frac{1}{6} k_{i j k} b^{i} b^{j} b^{k} m^{0} \equiv \rho_{0} .
\end{aligned}
$$

These are exactly (modulo some conventions) the on-shell values of the four-forms obtained in $[25,44]$ by dimensionally reducing the ten-dimensional Hodge duality relations between the type IIA RR field strengths.

Substituting (4.25) back into the bosonic Lagrangian (4.23) and (4.24), one obtains the scalar potential of the original theory which coincides with the well-known form of the type IIA RR flux potential [50,57],

$$
\begin{aligned}
V= & -\mathrm{e}^{-1} \mathcal{L}_{3 \text {-form }} \text { on-shell } \\
= & \mathrm{e}^{\hat{K}}\left[16 \mathrm{e}^{K} \rho_{0}^{2}+4 \mathrm{e}^{K} K^{i j} \rho_{i} \rho_{j}\right. \\
& \left.+\mathrm{e}^{-K} K_{i j} p^{i} p^{j}+\frac{1}{4}\left(m^{0}\right)^{2} \mathrm{e}^{-K}\right] .
\end{aligned}
$$

Note that upon this substitution the term (4.24) is no more a total derivative. Without the contribution of this term, the effective scalar potential would have a wrong (negative) sign. This is why we have kept track of the boundary terms in our construction all the time.

Note also that, if we substitute the on-shell values (4.25) of the four-forms $\mathcal{F}_{4}^{A}$ and $\tilde{\mathcal{F}}_{4 A}$ into the boundary Lagrangian (4.24), while still keeping the potentials $A^{m A}$ and $\tilde{A}_{A}^{m}$ offshell, upon some algebra we get

$$
\begin{aligned}
\hat{\mathcal{L}}_{\mathrm{bd}} & =2 e\left(\rho_{0}{ }^{*} \mathcal{F}_{4}^{0}+\rho_{i}{ }^{*} \mathcal{F}_{4}^{i}+p^{i *} \tilde{\mathcal{F}}_{4 i}+m^{0 *} \tilde{\mathcal{F}}_{40}\right) \\
& =2 \partial_{m}\left(e\left(m^{A} \tilde{A}_{A}^{m}-e_{A} A^{A m}\right)\right) .
\end{aligned}
$$

This boundary term is the same as the linear term of the effective Lagrangian obtained in [25] by the dimensional reduction of the democratic type IIA pseudo-action of [54]. It is a total derivative because of the use of the ten-dimensional Hodge duality relations between the lowerand higher-form RR field strengths, which, as we have already mentioned, are equivalent to the on-shell expressions (4.25) for the four-forms (see [25] for details). To perform the off-shell dimensional reduction one could use the fullfledged duality-symmetric action of type IIA supergravity constructed in [58]. In this way, in principle, one should get the four-dimensional Lagrangian (4.23) with the boundary term (4.24), which produces the constants $e_{A}$ and $m^{A}$ after one integrates out the 3 -forms.

\section{Conclusion}

In this paper we have shown how to construct globally and locally supersymmetric models with gauge three-forms, by dualizing more conventional theories with standard chiral multiplets and a superpotential of the form (1.1). In the dualization process, the coupling constants $e_{A}, m^{B}$ are promoted to (appropriate combinations of) expectation values of the field strengths $F_{4}^{A}=\mathrm{d} A_{3}^{A}, \tilde{F}_{A 4}=\mathrm{d} \tilde{A}_{A 3}$ associated with the three-form gauge fields $A_{3}^{A}, \tilde{A}_{A 3}$. The dual theory is manifestly supersymmetric and is constructed in terms of three-form multiplets which contain a complex scalar and one or two gauge three-forms as bosonic components, the latter replacing scalar auxiliary fields of the conventional chiral multiplets.

As an application, we applied our duality procedure to the four-dimensional effective theory describing the closed string sector of type IIA orientifold compactifications on Calabi-Yau three-folds with RR fluxes. In particular, we discussed the explicit form of the bosonic action for the scalar and three-form fields. By solving the equations of motion for the three-form fields we found the same on-shell values of their field strengths as those obtained by direct dimensional reduction [25] and the correct potential for the scalar fields [50].

Even though our approach is quite general, the application to more general string compactifications requires further work. First of all, in the type IIA models considered in Sect. 4 the tadpole condition does not directly concern the internal fluxes that are involved in the dualization. In more general IIA compactifications, for instance with a non-trivial $H_{3}$-flux, the tadpole condition would become relevant for the dualization procedure. The same is true for type IIB orientifold compactifications, which have a flux-induced superpotential [51-53,59,60] compatible with our general framework too. Also in these cases a non-trivial tadpole condition should be appropriately taken into account.

Another aspect that deserves further study is the inclusion of the open-string sector in the effective theory, which may be naturally incorporated in a three-form formulation [42-44]. It would be interesting to revisit this point in the manifestly supersymmetric framework provided in the present paper. Related questions concern its applications to M-theory and Ftheory compactifications, which can be considered as strong coupling limits of type IIA and IIB compactifications with back-reacting branes; see for instance $[61,62]$ for reviews.

In four dimensions, gauge three-forms couple to membranes that appear as domain-walls generating jumps of the 
value of the corresponding field strength, as e.g. in [4]. In the context of string/M-theory compactifications, these membranes correspond to higher-dimensional branes wrapping various cycles in the internal space and are crucial for the mechanisms of dynamical relaxation of the cosmological constant discussed, for instance, in $[9,10]$. Our formulation should be the starting point for revisiting these aspects at the level of a four-dimensional effective theory with manifest linearly realized supersymmetry, generalizing the results of $[8,12-14]$. Furthermore, in this same context and in the presence of spontaneously broken supersymmetry, our formulation should be related, at low energies, to models with non-linearly realized local supersymmetry as the ones introduced in [15]. It would be interesting to elucidate this relation. More generically, it would be worth using this general framework to construct and study supersymmetric extensions of various models based on gauge three-forms, as for instance those discussed in [1,3-7,11,16-19,22-26].

Acknowledgements We thank Igor Bandos, Massimo Bianchi, Sergei Kuzenko and Irene Valenzuela for useful discussions and comments. Work of F.F., S.L. and L.M. was partially supported by the Padua University Project CPDA144437. Work of D.S. was supported in part by the Australian Research Council Project No. DP160103633 and by the Russian Science Foundation grant 14-42-00047 in association with Lebedev Physical Institute.

Open Access This article is distributed under the terms of the Creative Commons Attribution 4.0 International License (http://creativecomm ons.org/licenses/by/4.0/), which permits unrestricted use, distribution, and reproduction in any medium, provided you give appropriate credit to the original author(s) and the source, provide a link to the Creative Commons license, and indicate if changes were made.

Funded by SCOAP ${ }^{3}$.

\section{A Component structure of $\mathcal{N}=1$ superfields}

In this appendix we collect some useful formulas on the component structure of the multiplets considered in the present paper. We mostly follow notation and conventions of [45].

The chiral multiplet $\Phi$ is defined by the condition

$\bar{D}_{\dot{\alpha}} \Phi=0$.

Its component expansion is

$$
\begin{gathered}
\Phi=\varphi+\sqrt{2} \theta \psi+\theta^{2} F+i \theta \sigma^{m} \bar{\theta} \partial_{m} \varphi \\
-\frac{i}{\sqrt{2}} \theta^{2} \partial_{m} \psi \sigma^{m} \bar{\theta}+\frac{1}{4} \theta^{2} \bar{\theta}^{2} \square \varphi,
\end{gathered}
$$

where $\varphi$ and $F$ are complex scalar fields and $\psi$ is a Weyl spinor. The independent bosonic components of $\Phi$ are defined as follows:

$$
\begin{aligned}
\Phi \mid & =\varphi, \\
-\frac{1}{4} D^{2} \Phi \mid & =F,
\end{aligned}
$$

where the vertical line means that the quantity is evaluated at $\theta=\bar{\theta}=0$. The real scalar multiplet $U$ has the following component structure:

$$
\begin{aligned}
U= & u+i \theta \chi-i \bar{\theta} \bar{\chi}+i \theta^{2} \bar{\varphi}-i \bar{\theta}^{2} \varphi+2 \theta \sigma^{m} \bar{\theta} A_{m} \\
& +i \theta^{2} \bar{\theta}\left(\bar{\lambda}+\frac{i}{2} \bar{\sigma}^{m} \partial_{m} \chi\right)-i \bar{\theta}^{2} \theta\left(\lambda+\frac{i}{2} \sigma^{m} \partial_{m} \bar{\chi}\right) \\
& -\theta^{2} \bar{\theta}^{2}\left(D+\frac{1}{4} \square u\right),
\end{aligned}
$$

where $u$ and $D$ are real scalar fields, $\varphi$ is a complex scalar field, $A_{m}$ is a real vector field and $\chi$ and $\lambda$ are Weyl spinors. The independent bosonic components of $U$ are defined as follows:

$$
\begin{aligned}
U \mid & =u, \\
-\frac{1}{8} \bar{\sigma}_{m}^{\dot{\alpha} \alpha}\left[D_{\alpha}, \bar{D}_{\dot{\alpha}}\right] U \mid & =A_{m}, \\
\frac{i}{4} D^{2} U \mid & =\bar{\varphi}, \\
\frac{1}{16} D^{2} \bar{D}^{2} U \mid & =-D+i \partial^{m} A_{m} .
\end{aligned}
$$

The real linear multiplet $L$ is a real multiplet which, in addition, satisfies the condition

$$
D^{2} L=0, \quad \bar{D}^{2} L=0 .
$$

The component expansion of $L$ has the form

$$
\begin{aligned}
L= & l+i \theta \eta-i \bar{\theta} \bar{\eta}+\frac{1}{3} \theta \sigma_{m} \bar{\theta} \varepsilon^{m n p q} \partial_{[n} \Lambda_{p q]} \\
& +\frac{1}{2} \theta^{2} \bar{\theta} \bar{\sigma}^{m} \partial_{m} \eta-\frac{1}{2} \theta \bar{\theta}^{2} \sigma^{m} \partial_{m} \bar{\eta}-\frac{1}{4} \theta^{2} \bar{\theta}^{2} \square l
\end{aligned}
$$

where $l$ is a real scalar, $\Lambda_{m n}$ is a rank 2 antisymmetric tensor and $\eta$ is a Weyl spinor.

The bosonic components of $L$ are defined through the projections

$$
\begin{aligned}
L \mid & =l, \\
\frac{1}{2} \bar{\sigma}^{m \dot{\alpha} \alpha}\left[D_{\alpha}, \bar{D}_{\dot{\alpha}}\right] L \mid & =-\frac{2}{3} \varepsilon^{m n p q} \partial_{[n} \Lambda_{p q]} .
\end{aligned}
$$

The complex linear multiplet $\Sigma$ satisfies the condition

$\bar{D}^{2} \Sigma=0$

Its $\theta$-expansion is

$$
\begin{aligned}
\Sigma= & \sigma+\theta \psi+\sqrt{2} \bar{\theta} \bar{\rho}-\frac{1}{2} \theta \sigma_{m} \bar{\theta} C^{m}+\theta^{2} \bar{s}+\theta^{2} \bar{\theta} \bar{\xi} \\
& -\frac{i}{\sqrt{2}} \bar{\theta}^{2} \theta \sigma^{m} \partial_{m} \bar{\rho}+\theta^{2} \bar{\theta}^{2}\left(\frac{i}{4} \partial_{m} C^{m}-\frac{1}{4} \square \sigma\right) .
\end{aligned}
$$

Here $\sigma$ and $\bar{s}$ are complex scalars, $\rho, \psi$ and $\xi$ are Weyl spinors and $C^{m}$ is a complex vector which is Hodge dual to the three-form 
$C^{m}=\frac{1}{3 !} \varepsilon^{m n p q} C_{n p q}$.

The bosonic components of $\Sigma$ are defined by the projections

$$
\begin{aligned}
\Sigma \mid & =\sigma, \\
\frac{1}{2} \bar{\sigma}^{m \dot{\alpha} \alpha}\left[D_{\alpha}, \bar{D}_{\dot{\alpha}}\right] \Sigma \mid & =C^{m}, \\
-\frac{1}{4} D^{2} \Sigma \mid & =\bar{s}, \\
\frac{1}{16} D^{2} \bar{D}^{2} \Sigma \mid & =0, \\
\frac{1}{16} \bar{D}^{2} D^{2} \Sigma \mid & =\frac{i}{2} \partial_{m} C^{m} .
\end{aligned}
$$

\section{B Note on three-forms, scalar potentials and boundary terms}

In this appendix we illustrate the dualization procedure with two simple examples: first we will consider a purely bosonic Lagrangian of a single gauge three-form and then we will examine the case of a Lagrangian with a single complex linear multiplet.

Let us consider a real three-form with couplings described by the Lagrangian

$$
\begin{aligned}
\mathcal{L} & =K^{\prime \prime}(\varphi)\left(\frac{1}{3 !} \partial_{m} \varepsilon^{m n p q} A_{n p q}\right)^{2} \\
& +W^{\prime}(\varphi)\left(\frac{1}{3 !} \partial_{m} \varepsilon^{m n p q} A_{n p q}\right)
\end{aligned}
$$

where $K^{\prime \prime}(\varphi)$ and $W^{\prime}(\varphi)$ are real functions of the scalar fields $\varphi$, denoted in this way to be reminiscent of the structure of supersymmetric chiral field models. To further simplify the formulas, let us replace $A_{n p q}$ with its Hodge-dual vector field

$A^{m}=\frac{1}{3 !} \varepsilon^{m n p q} A_{n p q}$,

so that (B.1) becomes

$$
\mathcal{L}=K^{\prime \prime}(\varphi)\left(\partial_{m} A^{m}\right)^{2}+W^{\prime}(\varphi)\left(\partial_{m} A^{m}\right)
$$

Note that the gauge invariance of the three-form becomes an invariance of the action under the transformation of the one-form $A_{1} \rightarrow A_{1}+{ }^{*} \mathrm{~d} \Lambda_{2}$, where $A_{1}=A_{m} \mathrm{~d} x^{m}$.

We wish to integrate out $A^{m}$ to find the contribution to the scalar potential. To perform a consistent variation of the action with respect to the three-form, one should introduce an appropriate boundary term of the form $[4,6]$

$$
\mathcal{L}_{\mathrm{bd}}=-\partial_{m}\left(\left(W^{\prime}+2 K^{\prime \prime} \partial_{n} A^{n}\right) A^{m}\right) .
$$

Then the equations of motion for the three-form (which are unaffected by the boundary terms) give

$\partial_{m} A^{m}=-\frac{W^{\prime}+r}{2 K^{\prime \prime}}$,

where $r$ is a real integration constant. Substituting (B.5) into (B.3) + (B.4) we get the following Lagrangian which provides the potential for the scalar fields $\varphi$ :

$\mathcal{L}=-\frac{\left(r+W^{\prime}\right)^{2}}{4 K^{\prime \prime}}$

There is an alternative way to integrate out the three-form without the need to introduce the boundary terms. We can rewrite (B.3) by using a Lagrange multiplier scalar $\alpha$ and an auxiliary field $F$

$\mathcal{L}=K^{\prime \prime} F^{2}+W^{\prime} F+\alpha F+A^{m} \partial_{m} \alpha$.

By varying $\alpha$ in (B.7) with the boundary condition $\left.\delta \alpha\right|_{\text {bd }}=$ 0 we get $F=\partial_{m} A^{m}$ and then back (B.3). Now $A^{m}$ is a Lagrange multiplier and we can consistently integrate it out without the need of additional boundary terms thus getting

$\alpha=r$,

where $r$ is a real constant related to the on-shell value of $F_{4}=d A_{3}$. Now we have

$\mathcal{L}=K^{\prime \prime} F^{2}+\left(r+W^{\prime}\right) F$

and once we integrate out the scalar $F$ we find (B.6), which produces a positive definite contribution to the scalar potential (if $K^{\prime \prime}>0$ ).

On the other hand, this dualization procedure provides a systematic way to get the boundary term (B.4), which is necessary to make the variation of the Lagrangian (B.1) consistent. To do this we should reverse the dualization procedure starting from the Lagrangian (B.7). The variation of the Lagrangian (B.7) with respect to the auxiliary field gives

$\begin{aligned} \delta_{\alpha} \mathcal{L} & =\delta \alpha\left(F-\partial_{m} A^{m}\right)+\partial_{m}\left(A^{m} \delta \alpha\right), \\ \delta_{F} \mathcal{L} & =\left(2 K^{\prime \prime} F+\alpha+W^{\prime}\right) \delta F .\end{aligned}$

Imposing the boundary conditions

$\left.\delta \alpha\right|_{\mathrm{bd}}=0,\left.\quad \delta F\right|_{\mathrm{bd}}=0$,

and setting the variations to zero we get

$\alpha=-2 K^{\prime \prime} F-W^{\prime}, \quad F=\partial_{m} A^{m}$. 
Plugging (B.12) back into the Lagrangian (B.7), we get

$\mathcal{L}=K^{\prime \prime}\left(\partial_{m} A^{m}\right)^{2}+W^{\prime} \partial_{m} A^{m}-\partial_{m}\left(A^{m}\left(W^{\prime}+2 K^{\prime \prime} \partial_{n} A^{n}\right)\right)$,

which reproduces the boundary term (B.4).

Let us now consider an example which shows how a Lagrangian of the form (B.3) can be obtained by the direct computation of the bosonic components of a superspace Lagrangian of the form discussed in Sect. 2. Let us consider the following Lagrangian for a single chiral multiplet $\Phi$ (whose component structure was given in (A.3)):

$\mathcal{L}=\int \mathrm{d}^{4} \theta \Phi \bar{\Phi}+\left(\int \mathrm{d}^{2} \theta(c \Phi+W(\Phi))+\right.$ c.c. $)$,

with $c$ being a complex constant. In order to make the auxiliary field $F$ of $\Phi$ dynamical, we promote the complex constant $c$ to a chiral superfield $X$ and add a new term which contains the complex linear superfield $\Sigma$

$$
\begin{aligned}
\mathcal{L}= & \int \mathrm{d}^{4} \theta \Phi \bar{\Phi}+\left(\int \mathrm{d}^{2} \theta(X \Phi+W(\Phi))+\text { c.c. }\right) \\
& +\left[\int \mathrm{d}^{2} \theta\left(-\frac{1}{4} \bar{D}^{2}\right)(\bar{X} \Sigma)+\text { c.c. }\right] .
\end{aligned}
$$

Using the expansions of the superfields in component fields given in Appendix A and focusing on the bosonic components only, we get from (B.15) the following part of the component Lagrangian which contains the auxiliary fields $F$ and $\bar{F}$ :

$\mathcal{L}_{F}=F \bar{F}+\left(W^{\prime} F+\alpha F+\frac{i}{2} C^{m} \partial_{m} \alpha+\right.$ c.c. $)$,

where $\alpha=X \mid$ and, as usual, the vector field $C^{m}$ is the dual of a three-form. This is a complexified version of the Lagrangian (B.7).

To obtain the dual Lagrangian for the fields $C^{m}$ we vary (B.16) with respect to $\alpha$ and $F$, and get the equations of motion

$F=\frac{i}{2} \partial_{m} C^{m}$,

$\alpha=-\bar{F}-W^{\prime}$.

Plugging them back into (B.16), we get

$\mathcal{L}=\frac{1}{4}\left(\partial_{n} C^{n}\right)\left(\partial_{m} \bar{C}^{m}\right)+\left(\frac{i}{2} W^{\prime} \partial_{n} C^{n}+\right.$ c.c. $)+\mathcal{L}_{\mathrm{bd}}$,

with the boundary term Lagrangian having the required form

$\mathcal{L}_{\mathrm{bd}}=\frac{i}{2} \partial_{m}\left(\left(\frac{i}{2} \partial_{n} C^{n}-W^{\prime}\right) C^{m}\right)+c . c$.

\section{Properties of the Kähler potential and superpotential of type IIA compactifications with RR fluxes}

Here we give some useful expressions that we used for the analysis of the effective four-dimensional theory associated with the example of type IIA flux compactification in Sect. 4.

The $K$ part (4.5) of the Kähler potential (4.3) of the model under consideration is

$K=-\log 8 k$,

where

$k=\frac{1}{3 !} k_{i j k} v^{i} v^{j} v^{k}, \quad k_{i j} \equiv k_{i j k} v^{k}, \quad k_{i} \equiv k_{i j k} v^{j} v^{k}$

and $k_{i j k}$ is the triple intersection number of the $\mathrm{CY}_{3}$ manifold.

Defining $K_{i} \equiv \frac{\partial K}{\partial \varphi^{i}}$ and $K_{i j} \equiv \frac{\partial^{2} K}{\partial \varphi^{i} \partial \bar{\varphi}^{j}}$, we have

$$
\begin{aligned}
K_{i} & =-\frac{k_{i}}{4 k}=-2 k_{i} \mathrm{e}^{K}, \\
K_{i j} & =-\frac{1}{4 k}\left(k_{i j}-\frac{k_{i} k_{j}}{4 k}\right), \\
K^{i j} \equiv\left(K_{i j}\right)^{-1} & =-4 k\left(k^{i j}-\frac{v^{i} v^{j}}{2 k}\right),
\end{aligned}
$$

and

$K^{i j} K_{j}=-2 v^{i}, \quad K_{i j} v^{j}=-\frac{1}{2} K_{i}, \quad K_{i} v^{i}=-\frac{3}{2}$.

From (4.12), upon gauge-fixing $\mathcal{Z}_{0}=1$, we get the following components of the imaginary and the real parts of the holomorphic matrix $\mathcal{G}_{A B}$ (2.38):

$$
\begin{aligned}
& \mathcal{M}_{00}=-2 k+k_{i j} b^{i} b^{j}, \quad \mathcal{M}_{0 i}=-k_{i j} b^{j}=-\mathcal{M}_{i j} b^{j}, \\
& \mathcal{M}_{i j}=k_{i j}, \\
& \mathcal{N}_{00}=\frac{1}{3} k_{i j k} b^{i} b^{j} b^{j}-k_{i} b^{i}, \quad \mathcal{N}_{0 i}=\frac{1}{2}\left(k_{i}-k_{i j k} b^{j} b^{k}\right), \\
& \mathcal{N}_{i j}=k_{i j k} b^{k} .
\end{aligned}
$$

The inverse matrix $\mathcal{M}^{A B}$ has the following components:

$\mathcal{M}^{00}=-\frac{1}{2 k}, \quad \mathcal{M}^{0 i}=-\frac{1}{2 k} b^{i}, \quad \mathcal{M}^{i j}=k^{i j}-\frac{1}{2 k} b^{i} b^{j}$.

\section{References}

1. M.J. Duff, P. van Nieuwenhuizen, Quantum inequivalence of different field representations. Phys. Lett. B 94, 179-182 (1980)

2. A. Aurilia, H. Nicolai, P.K. Townsend, Hidden constants: the theta parameter of QCD and the cosmological constant of $\mathrm{N}=8$ supergravity. Nucl. Phys. B 176, 509-522 (1980) 
3. S.W. Hawking, The cosmological constant is probably zero. Phys. Lett. B 134, 403 (1984)

4. J.D. Brown, C. Teitelboim, Dynamical neutralization of the cosmological constant. Phys. Lett. B 195, 177-182 (1987)

5. J.D. Brown, C. Teitelboim, Neutralization of the cosmological constant by membrane creation. Nucl. Phys. B 297, 787-836 (1988)

6. M.J. Duff, The cosmological constant is possibly zero, but the proof is probably wrong. Phys. Lett. B 226, 36 (1989) [Conf. Proc. C 8903131, 403 (1989)]

7. M.J. Duncan, L.G. Jensen, Four forms and the vanishing of the cosmological constant. Nucl. Phys. B 336, 100-114 (1990)

8. B.A. Ovrut, D. Waldram, Membranes and three form supergravity. Nucl. Phys. B 506, 236-266 (1997). arXiv:hep-th/9704045

9. R. Bousso, J. Polchinski, Quantization of four form fluxes and dynamical neutralization of the cosmological constant. JHEP 06, 006 (2000). arXiv:hep-th/0004134

10. J.L. Feng, J. March-Russell, S. Sethi, F. Wilczek, Saltatory relaxation of the cosmological constant. Nucl. Phys. B 602, 307-328 (2001). arXiv:hep-th/0005276

11. Z.C. Wu, The cosmological constant is probably zero, and a proof is possibly right. Phys. Lett. B 659, 891-893 (2008). arXiv:0709.3314 [gr-qc]

12. I.A. Bandos, C. Meliveo, Superfield equations for the interacting system of $\mathrm{D}=4 \mathrm{~N}=1$ supermembrane and scalar multiplet. Nucl. Phys. B 849, 1-27 (2011). arXiv:1011.1818 [hep-th]

13. I.A. Bandos, C. Meliveo, Three form potential in (special) minimal supergravity superspace and supermembrane supercurrent. J. Phys. Conf. Ser. 343, 012012 (2012). arXiv:1107.3232 [hep-th]

14. I.A. Bandos, C. Meliveo, Supermembrane interaction with dynamical $\mathrm{D}=4 \mathrm{~N}=1$ supergravity. Superfield Lagrangian description and spacetime equations of motion. JHEP 08, 140 (2012). arXiv: 1205.5885 [hep-th]

15. F. Farakos, A. Kehagias, D. Racco, A. Riotto, Scanning of the supersymmetry breaking scale and the gravitino mass in supergravity. JHEP 06, 120 (2016). arXiv:1605.07631 [hep-th]

16. G. Dvali, Three-form gauging of axion symmetries and gravity. arXiv:hep-th/0507215

17. G. Dvali, Large hierarchies from attractor vacua. Phys. Rev. D 74, 025018 (2006). arXiv:hep-th/0410286

18. G. Dvali, A vacuum accumulation solution to the strong $\mathrm{CP}$ problem. Phys. Rev. D 74, 025019 (2006). arXiv:hep-th/0510053

19. G. Dvali, S. Folkerts, A. Franca, How neutrino protects the axion. Phys. Rev. D 89(10), 105025 (2014). arXiv:1312.7273 [hep-th]

20. G. Dvali, L. Funcke, Small neutrino masses from gravitational $\theta$ term. Phys. Rev. D 93(11), 113002 (2016). doi:10.1103/PhysRevD. 93.113002 [arXiv:1602.03191 [hep-ph]]

21. G. Dvali, L. Funcke, Domestic Axion. arXiv:1608.08969 [hep-ph]

22. N. Kaloper, L. Sorbo, A natural framework for Chaotic inflation. Phys. Rev. Lett. 102, 121301 (2009). arXiv:0811.1989 [hep-th]

23. N. Kaloper, A. Lawrence, L. Sorbo, An ignoble approach to large field inflation. JCAP 1103, 023 (2011). arXiv:1101.0026 [hep-th]

24. F. Marchesano, G. Shiu, A.M. Uranga, F-term axion monodromy inflation. JHEP 09, 184 (2014). arXiv:1404.3040 [hep-th]

25. S. Bielleman, L.E. Ibanez, I. Valenzuela, Minkowski 3-forms, flux string vacua, axion stability and naturalness. JHEP 12, 119 (2015). arXiv: 1507.06793 [hep-th]

26. E. Dudas, Three-form multiplet and Inflation. JHEP 12, 014 (2014). arXiv: 1407.5688 [hep-th]

27. I. Valenzuela, Backreaction issues in axion monodromy and minkowski 4-forms. arXiv:1611.00394 [hep-th]

28. K.S. Stelle, P.C. West, Minimal auxiliary fields for supergravity. Phys. Lett. B 74, 330 (1978)

29. V. Ogievetsky, E. Sokatchev, Structure of supergravity group. Phys. Lett. B 79, 222 (1978) [Czech. J. Phys. B 29, 68 (1979)]
30. V. Ogievetsky, E. Sokatchev, Equation of motion for the axial gravitational superfield. Sov. J. Nucl. Phys. 32, 589 (1980) [Yad. Fiz.32,1142(1980)]

31. S.J. Gates Jr., Super P-form gauge superfields. Nucl. Phys. B 184, 381-390 (1981)

32. S.J. Gates Jr., W. Siegel, Variant superfield representations. Nucl. Phys. B 187, 389-396 (1981)

33. I.L. Buchbinder, S.M. Kuzenko, Quatization of the classically equivalent theories in the superspace of simple supergravity and quantum equivalence. Nucl. Phys. B 308, 162-190 (1988)

34. P. Binetruy, F. Pillon, G. Girardi, R. Grimm, The three form multiplet in supergravity. Nucl. Phys. B 477, 175-202 (1996). arXiv:hep-th/9603181

35. S.M. Kuzenko, S.A. McCarthy, On the component structure of $\mathrm{N}=1$ supersymmetric nonlinear electrodynamics. JHEP 05, 012 (2005). arXiv:hep-th/0501172

36. H. Nishino, S. Rajpoot, Alternative auxiliary fields for chiral multiplets. Phys. Rev. D 80, 127701 (2009)

37. M.J. Duff, S. Ferrara, Four curious supergravities. Phys. Rev. D 83, 046007 (2011). arXiv: 1010.3173 [hep-th]

38. K. Groh, J. Louis, J. Sommerfeld, Duality and couplings of 3form-multiplets in $\mathrm{N}=1$ supersymmetry. JHEP 05, 001 (2013). arXiv:1212.4639 [hep-th]

39. I. Bandos, M. Heller, S.M. Kuzenko, L. Martucci, D. Sorokin, The Goldstino brane, the constrained superfields and matter in $\mathcal{N}=1$ supergravity. JHEP 11, 109 (2016). arXiv:1608.05908 [hep-th]

40. S. Aoki, T. Higaki, Y. Yamada, R. Yokokura, Abelian tensor hierarchy in 4D $\mathcal{N}=1$ conformal supergravity. JHEP 09, 148 (2016). arXiv: 1606.04448 [hep-th]

41. E.I. Buchbinder, S.M. Kuzenko, Three-form multiplet and supersymmetry breaking. arXiv:1705.07700 [hep-th]

42. T.W. Grimm, D. Vieira Lopes, The $\mathrm{N}=1$ effective actions of Dbranes in Type IIA and IIB orientifolds. Nucl. Phys. B 855, 639-694 (2012). arXiv:1104.2328 [hep-th]

43. M. Kerstan, T. Weigand, The effective action of D6-branes in $\mathrm{N}$ = 1 type IIA orientifolds. JHEP 06, 105 (2011). arXiv:1104.2329 [hep-th]

44. F. Carta, F. Marchesano, W. Staessens, G. Zoccarato, Open string multi-branched and Kähler potentials. JHEP 09, 062 (2016). arXiv:1606.00508 [hep-th]

45. J. Wess, J. Bagger, Supersymmetry and supergravity (Princeton, USA: Princeton University Press, 1992), p. 259

46. P.S. Howe, R.W. Tucker, Scale invariance in superspace. Phys. Lett. B 80, 138-140 (1978)

47. S. Gates, M.T. Grisaru, M. Rocek, W. Siegel, Superspace or one thousand and one lessons in supersymmetry. Front. Phys. 58, 1-548 (1983). arXiv:hep-th/0108200

48. I. Buchbinder, S. Kuzenko, Ideas and methods of supersymmetry and supergravity: a walk through superspace (IOP, Bristol, UK, 1995), p. 640

49. D.Z. Freedman, A. Van Proeyen, Supergravity (Cambridge University Press, Cambridge, 2012)

50. T.W. Grimm, J. Louis, The Effective action of type IIA Calabi-Yau orientifolds. Nucl. Phys. B 718, 153-202 (2005). arXiv:hep-th/0412277

51. S. Gukov, C. Vafa, E. Witten, CFT's from Calabi-Yau four folds. Nucl. Phys. B 584, 69-108 (2000). arXiv:hep-th/9906070 [hep-th] [Erratum: Nucl. Phys.B608,477(2001)]

52. S. Gukov, Solitons, superpotentials and calibrations. Nucl. Phys. B 574, 169-188 (2000). arXiv:hep-th/9911011

53. T.R. Taylor, C. Vafa, $R$ R flux on Calabi-Yau and partial supersymmetry breaking. Phys. Lett. B 474, 130-137 (2000). arXiv:hep-th/9912152

54. E. Bergshoeff, R. Kallosh, T. Ortin, D. Roest, A. Van Proeyen, New formulations of $\mathrm{D}=10$ supersymmetry and $\mathrm{D} 8$ - O8 domain walls. Class. Quant. Grav. 18, 3359-3382 (2001). arXiv:hep-th/0103233 
55. P.C. West, E(11) and M theory. Class. Quant. Grav. 18, 4443-4460 (2001). arXiv:hep-th/0104081

56. A. Kleinschmidt, I. Schnakenburg, P.C. West, Very extended KacMoody algebras and their interpretation at low levels. Class. Quant. Grav. 21, 2493-2525 (2004). arXiv:hep-th/0309198

57. J. Louis, A. Micu, Type 2 theories compactified on Calabi-Yau threefolds in the presence of background fluxes, Nucl. Phys. B 635, 395-431 (2002). arXiv:hep-th/0202168

58. I.A. Bandos, A.J. Nurmagambetov, D.P. Sorokin, Various faces of type IIA supergravity, Nucl. Phys. B 676, 189-228 (2004). arXiv:hep-th/0307153

59. S.B. Giddings, S. Kachru, J. Polchinski, Hierarchies from fluxes in string compactifications. Phys. Rev. D 66, 106006 (2002). arXiv:hep-th/0105097
60. T.W. Grimm, J. Louis, The effective action of $\mathrm{N}=1$ Calabi-Yau orientifolds, Nucl. Phys. B 699, 387-426 (2004). arXiv:hep-th/0403067 [hep-th]

61. B.S. Acharya, S. Gukov, M theory and singularities of exceptional holonomy manifolds, Phys. Rept. 392, 121-189 (2004). arXiv:hep-th/0409191 [hep-th]

62. F. Denef, Les Houches Lectures on Constructing String Vacua, in String theory and the real world: From particle physics to astrophysics. Proceedings, Summer School in Theoretical Physics, 87th Session, Les Houches, France, 2008, pp. 483-610. arXiv:0803.1194 [hep-th] 